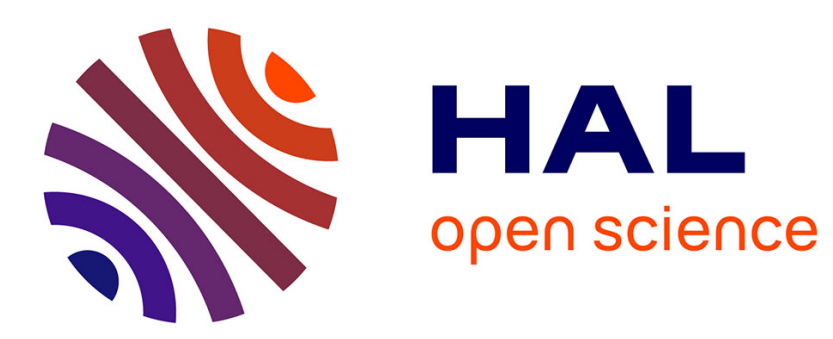

\title{
Turbulent structures of buoyant jet in cross-flow studied through large-eddy simulation
}

Carlo Cintolesi, Andrea Petronio, Vincenzo Armenio

\section{To cite this version:}

Carlo Cintolesi, Andrea Petronio, Vincenzo Armenio. Turbulent structures of buoyant jet in cross-flow studied through large-eddy simulation. Environmental Fluid Mechanics, 2018, 10.1007/s10652-0189629-1. hal-02070287

\section{HAL Id: hal-02070287 \\ https://hal.science/hal-02070287}

Submitted on 17 Mar 2019

HAL is a multi-disciplinary open access archive for the deposit and dissemination of scientific research documents, whether they are published or not. The documents may come from teaching and research institutions in France or abroad, or from public or private research centers.
L'archive ouverte pluridisciplinaire HAL, est destinée au dépôt et à la diffusion de documents scientifiques de niveau recherche, publiés ou non, émanant des établissements d'enseignement et de recherche français ou étrangers, des laboratoires publics ou privés. 


\title{
Turbulent structures of buoyant jet in cross-flow studied through large-eddy simulation
}

\author{
Carlo Cintolesi • Andrea Petronio . \\ Vincenzo Armenio
}

Received: date / Accepted: date

\begin{abstract}
In the present paper we study buoyant (plume) and non-buoyant (jet) fluid injection in a neutrally stratified uniform cross-flow. Both cases are of practical importance in environmental fluid mechanics. The study is carried out numerically, using highly resolved large-eddy simulation in conjunction with the Lagrangian dynamic sub-grid scale model for both momentum and scalar transport equations. The velocity ratio is $\kappa=8$. In the plume case, the Froude number is $F=10$, such to allow the use of the Boussinesq approximation. The simulations are successfully validated against experimental data and well established semiempirical relations. The study shows the existence of three different regions as regards the plume evolution, each of them characterised by different peculiarities: in momentum-buoyancy region the plume exhibits an almost steady cylindrical shape with relative small turbulence structures; in deflection region the plume is deviated horizontally and a high shear rate is detected; in entrainment region the vortex pair develops, along with the sausage-like turbulent structure. The comparison between the plume and the jet case shows that the latter has a higher eccentricity while its trajectory height is sensibly lower. Also, the sausage-like structures are not present. Finally, an empirical formula for the jet trajectory is given, although its full validation will require additional studies.
\end{abstract}

Keywords Large-eddy simulation · buoyant jet in cross-flow · turbulence structures · OpenFOAM.

Carlo Cintolesi · Andrea Petronio

IEFLUIDS S.r.l., Piazzale Europa 1, I-34127 Trieste, Italy

E-mail: carlo.cintolesi@gmail.com, a.petronio@iefluids.com

Vincenzo Armenio

University of Trieste, Dept Engineering and Architecture, via A. Valerio 10, I-34127 Trieste, Italy

E-mail: armenio@dica.units.it 


\section{Contents}

1 Introduction . . . . . . . . . . . . . . . . . . . . . . . 2

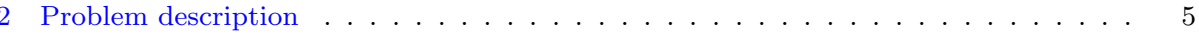

3 Simulation methodology and settings . . . . . . . . . . . . . . . . . . . . . 8

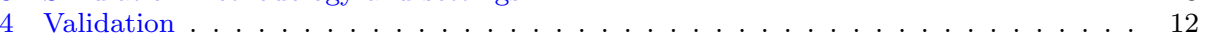

5 Mean flow analyses . . . . . . . . . . . . . . . . . . . . . . . . . . . . . 21

6 Second order statistics and turbulent structures . . . . . . . . . . . . . . . . . . . 25

7 Comparison with the non-buoyant case . . . . . . . . . . . . . . . . . . . . 32

8 Conclusions . . . . . . . . . . . . . . . . . . . . . . . . . . 35

NOTE: this is the final version of the manuscript accepted for publication. Reference to the published article:

- Carlo Cintolesi, Andrea Petronio, Vincenzo Armenio. Turbulent structures of buoyant jet in cross-flow studied through large-eddy simulation. Environmental Fluid Mechanics (2018). doi: dx.doi.org/10.1007/s10652-018-9629-1.

(C) 2018. This manuscript version is made available under the Creative Commons CC-BY-NCND 4.0 license.

\section{Introduction}

The study of a jet released into a cross-flow is of great relevance for a wide range of engineering problems, including: the pollutants dispersed by chimneys in the low atmosphere or from pipes in rivers and water bodies, the smoke generated by large-scale fire events, the combustor design, the optimised arrangement of the ship exhaust funnels. In all these examples, the accurate prediction of the jet evolution is of crucial importance for both environmental impact mitigation and devices optimisation. Particularly, the attention is generally focused on the trajectory followed by the jet, how the jet entrains the surrounding flow, the mass dispersion and the mixing efficiency of the system. Despite the large application range, the behaviour of such physical systems is not yet fully understood and the research on this topic is still ongoing.

Specifically, large literature is present for the case of neutral jet in a crossflow (JICF), whereas comparatively less work has been developed for the buoyant counterpart. The literature review of Mahesh [32] reports several results regarding round jet in incompressible regime (that of interest in our investigation). Regarding the mean flow characteristics, it is underlined that there is not a clear consensus on jet trajectory definition and scaling; the entrainment is significantly larger in transverse jet than in regular jet; the scalar mixing is qualitatively different in the near and far field, and it seems sensitive to jet-exit conditions as well as to near flow behaviour (Smith and Mungal [40] and Moussa et al. [35]). It is interesting to notice that the mixing is less strongly linked to the jet Reynolds number in gas-phase jet than in liquid-phase one (Dimotakis [14]). Concerning the turbulent structures, the classical coherent vortex systems are examined (horseshoes, wake and shear-layer vortices, and the counter-rotating vortex pair) pointing out that different structures arise when the velocity ratio between jet and cross-flow is less than one (Camussi et al. [3]). In such a regime, the jet-flow interaction is dominated by the hairpin-vortices and the flow exhibits strong oscillations due to 
periodic shear-layer instability. The author also marks that the Reynolds average simulations of JICF generally lead to simplistic prediction, because of the nonequilibrium nature of the turbulent flow.

Buoyancy introduces an additional free parameter to the system since in the present case the fate of the jet is ruled by momentum excess as well as by heat/concentration flux at the emission section. Different configurations have been studied in the past decades, both experimentally and numerically, for various combination of jet direction and ambient flow properties. Here we focus on round buoyant jet in cross-flow (BJCF) with uniform and non-stratified ambient flow since it is the case herein investigated.

Several experimental studies on the BJCF can be found in literature. One of the first work on this subject is that of Fan [16]. Two classes of buoyant jet (henceforward plume) problems were investigated: inclined round jet in stratified flow and round jet in a uniform and homogeneous cross stream. A number of experiments have been performed and analysed changing the initial and boundary conditions; the result is a complete database of experimental data. This is one of the references of the present investigation. Gaskin [21] performed a similar study, analysing a plume in parallel-flow and in stationary fluid. More recently, Meftah et al. [33] investigated a vertical round BJCF, focusing on the effect of crossflow turbulence generated by a rough bottom boundary on the structure of the plume. It turns out that the roughness induced significant modifications not only in the near-boundary field but also in the whole plume trajectory. The influence of turbulent cross-flow was studied by Huq and Stewart [25]. They pointed out that the plume entrainment and dilution rates experience a significant increase even for low turbulence (33\% higher dilution rate for weak turbulent cross-flow), eventually leading to a lower plume trajectory. Keramarisa and Pechlivanidis [27] focused on the effects of buoyancy force on plume behaviour. Their results show that the presence of buoyancy force strongly affects the flow dynamics of the system. Hence, neglecting of buoyancy in a large number of practical applications is an oversimplification that can lead to incorrect results.

Regarding LES of BJCF, a large part of the studies focusses on plume in stratified flows because of environmental applications: among the others we mention Devenish et al. [13] who analysed a buoyant jet from a circular source; Cunningham et al. [9] who investigated an archetypal case for buoyant plumes from wild-land fires using a compressible model; Lavelle [29] who studied the buoyancy-driven plumes in rotating and stratified cross-flows.

Numerical studies of boundary jet in uniform and non-stratified cross-flow are more limited in literature. Yuan and Street [44] investigated a jet in cross-flow in absence of buoyancy force, using a dynamic Smagorinsky Sub-Grid Scale (SGS) model. A set of simulations was performed and their results compared with empirical correlation laws. In a companion paper, Yuan et al. [45] analysed with details the coherent structures that arise in the region of the jet exit. The authors suggested that the well-known counter-rotating vortex pair present in the jet entrainment region is generated by the hanging vortices arising in the region near the jet exit. Devenish et al. [13] presented a LES of purely buoyant plume in a stratified environment. The SGS model was of the eddy viscosity type, with the sub-grid scale viscosity computed as a function of mixing length and Richardson number. The results were compared with plume models based on integral formulation of conservation laws; a loss in accuracy was detected for low-intensity cross-flow and 
some modifications to the model were proposed. Ma et al. [31] studied a BJCF, paying particular attention to the reproduction of the bent-over region, i.e. the region where the cross-flow is sufficiently strong to deviate the plume horizontally and gave a qualitative picture of turbulent structures. In the work of de Wit et al. [11] the practical case of a dredging plume dispersed from a moving trialling suction hopper dredger was studied. After a theoretical analysis of the problem, the results were validated against experimental data and semi-empirical solutions. The compressible Navier-Stokes equations were solved with a wall-adapting local eddy viscosity model. A passive scalar was used to track the jet volume concentration. The solver has been tested in the previous paper of de Wit and van Rhee [10] where a prototype case was simulated and studied.

In BJCF cases, the first purpose is to predict the general behaviour of the plume, e.g. the trajectory and the dilution rate. For this reason, the development of semi-empirical relations and integral models is a point of interest. Chu and Goldberg [5] provided a good introduction to this subject: a description of the non-dimensional parameters that govern the BJCF are reported, along with the analytical expression of the plume trajectory and its validation against experimental data. In the studies of Wright [42] and [43] the validity of the theoretical model that predicts trajectory and the empirical coefficients used are discussed, together with the different regimes observed for the buoyant jet (depending upon the relative magnitude of the momentum and buoyant length scale). Finally, the textbook of Lee and Chu [30] provided an updated review of the jet-integral descriptions of buoyant and non-buoyant jet in cross-flow mixing.

The present study is focused on the case of a BJCF injected perpendicularly to the main stream. Specifically, we study the turbulent structures and the physical mechanisms governing the buoyant jet in a cross-flow. We focus on a circular jet. Also, for comparison purposes we simulate an equivalent non-buoyant jet in the same cross-flow condition. A customised solver developed within the opensource software OpenFOAM performs the simulation. The environment fluid is non-stratified and the inlet flow is uniform. The system is characterised by largescale coherent structures and it is highly turbulent and efficient in mixing. The unsteadiness inherent in the problem makes the LES a better approach of simulation with respect to the Reynolds Average Navier-Stokes (RANS) simulations; however, this approach poses some difficulties. From a modelling point of view, an accurate turbulence model has to be adopted because the interaction between the two flows triggers non-isotropic turbulence mainly localised in the proximity of the plume. In this concern, the dynamic Lagrangian turbulent model is a stateof-the-art model for reproducing the physics undergoing in such systems; hence, it is adopted for the simulation. From a numerical side, attention needs to be paid to the discretisation schemes used because of the well-known problem of wiggle formation at the front side of the plume (see [11]). In particular, a careful choice of the scheme for advection term is a critical issue. Moreover, the presence of buoyancy force increases the complexity of the system and requires suitable boundary conditions at the domain outflow.

The paper is organised as follow: in section 2 the case geometry and semiempirical relations are presented; in section 3 the simulation methodology and settings are reported, along with the computational grid structure; in section 4 the validation is performed against experimental data and semi-empirical relations, a grid quality assessment is also addressed; in section 5 the first-order statistics are 


\begin{tabular}{|c|c|}
\hline Nomenclature & \\
\hline $\begin{array}{ll}\text { Latin symbols } \\
\mathrm{S}_{i k} & \text { fluctuation strain rate tensor } \\
B_{j 0} & \text { initial buoyancy flux } \\
c_{s} & \text { Smagorinsky constant for mo- } \\
& \text { mentum } \\
D & \text { diameter of cylindrical nozzle } \\
E_{\psi \psi} & \text { time power spectra of } \psi \\
g & \text { gravity acceleration magnitude } \\
k_{n} & \text { mass coefficient } \\
p & \text { dynamic pressure } \\
Q & \text { second invariant of velocity gra- } \\
& \text { dient tensor } \\
Q_{j 0} & \text { initial volume flux } \\
s_{j} & \text { plume active scalar (salinity) } \\
S_{i k} & \text { strain rate tensor } \\
u & \text { velocity magnitude } \\
z^{*} & \text { height where entrainment starts } \\
z_{B} & \text { height of buoyancy influence } \\
z_{M} & \text { height of momentum influence } \\
\text { Greek } & \text { symbols } \\
\alpha_{s} & \text { molecular salinity diffusivity } \\
\beta & \text { calibration parameter } \\
\beta_{s} & \text { volumetric contraction coeffi- } \\
& \text { cient of salinity }\end{array}$ & $\begin{array}{ll}\Delta & \text { filter/grid width } \\
\delta_{i k} & \text { Kronecker delta } \\
\epsilon & \text { dissipation rate of TKE } \\
\eta & \text { Kolmogorov length scale } \\
\nu & \text { molecular kinematic viscosity } \\
\rho & \text { space-time variable density } \\
\tau_{i k} & \text { SGS stress tensor } \\
\text { Non-dimensional numbers } \\
\kappa & \text { velocity ratio } \\
F & \text { Froude number } \\
R e & \text { Reynolds number } \\
S c & \text { Schmidt number } \\
\text { Subscripts and superscripts } \\
\langle\psi\rangle & \text { time averaged quantity } \\
\psi & \text { grid space filter } \\
\psi & \text { generic variable } \\
\psi^{\prime} & \text { variation from mean value } \\
\psi_{0} & \text { reference value } \\
\psi_{\text {SGS }} & \text { sub-grid scale quantity } \\
\psi_{c f} & \text { uniform cross-flow value } \\
\psi_{j 0} & \text { initial plume value } \\
\psi_{j} & \text { plume related quantity } \\
\widehat{\psi} & \text { test space filter }\end{array}$ \\
\hline
\end{tabular}

presented and discussed; in section 6 the second-order statistics and the turbulent structures are analysed; in section 7 an identical simulation where buoyancy is switched off are presented; in section 8 a summary of the results is given.

\section{Problem description}

The experiment of round buoyant jet in uniform cross-flow of homogeneous density proposed by Fan [16] is here reproduced. The experimental apparatus is composed as follow: the ambient fluid consists of a rectangular tank filled with fresh water (free surface at the top); a plume of salt water is injected from the top of the tank by a moving nozzle. In such a way, a sinking jet in a virtual uniform cross-flow is reproduced. The walls of the tank are considered sufficiently far to not influence the plume dynamics. Details on the measurements and the experiment settings can be found in see [16] and are not here repeated.

For a better visualisation, the BJCF is turned upside down and displayed as an ascending plume. The experimental data, photographs and graphs are simply turned upside down when compared with simulation results.

\subsection{Case geometry}

The geometry simulated is sketched in Figure 1. The uniform ambient cross-flow is oriented along the positive $x$-direction with magnitude $u_{c f}$, while the plume exits 


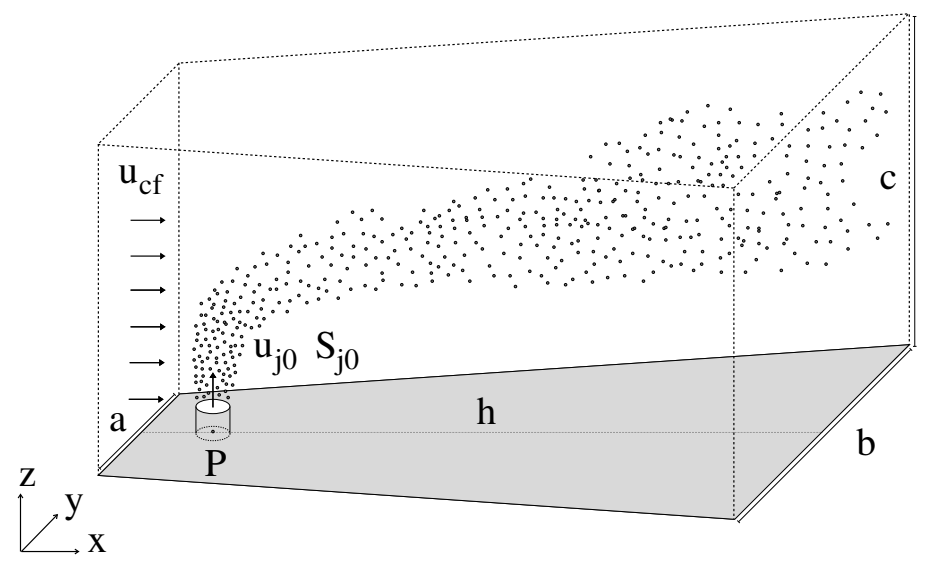

Fig. 1: Sketch of the geometry of the BJCF case under consideration. For better visualisation the cylindrical nozzle is not in scale.

from a cylindrical nozzle (placed at the bottom surface) in the positive $z$-direction with magnitude $u_{j 0}$. The plume has an initial salinity concentration $s_{j 0}$ at the nozzle exit; the ambient fluid has $s_{c f}$ at the inlet.

The nozzle has diameter and height $D=0.01 \mathrm{~m}$. The base central-point $P$ is located in the centre-line with respect to the $y$-direction, at a distance $2 D$ from the cross-flow inlet. Because the plume diffuses and becomes larger and larger in the stream-wise direction, the domain is enlarged at the outflow. Hence, it has the shape of a trapezoidal prism with the following dimensions: height of the prism $c=70 D$; trapezoid height $h=120 D$, minor edge $a=40 D$ and major edge $b=100 D$.

\subsection{Non-dimensional parameters}

The non-dimensional number that rules the BJCF is the plume Froude number (see Fan [16]), defined as the ratio of the cross-flow inertia to the plume motion:

$$
F=\frac{u_{j 0}}{\sqrt{\frac{\rho_{c f}-\rho_{j 0}}{\rho_{c f}} g D}}=\frac{u_{j 0}}{\sqrt{\beta_{s} \Delta s g D}}=\frac{u_{j 0}}{u_{b}},
$$

where $u_{b}=\sqrt{\beta_{s} \Delta s g D}$ is the buoyant velocity and the second equality is obtained adopting the Boussinesq approximation to model density variation. The global salinity variation is notated as $\Delta s=s_{j 0}-s_{c f}$ (see the nomenclature table for other symbols).

Other non-dimensional numbers are the Reynolds number (i.e. ratio of inertial forces to viscous forces):

$$
R e=\frac{u_{j 0} D}{\nu},
$$

and the velocity ratio:

$$
\kappa=\frac{u_{j 0}}{u_{c f}},
$$


which represents the relative strength of the jet to the cross stream.

Non-dimensional numbers analogous to the ones mentioned above are sometimes used in literature. For example, in de Wit et al. [11] the velocity ratio is defined as the square root of (3), and instead of the Froude number it is used the Richardson number that is the square of the reciprocal of (1).

In the following, lengths are made non-dimensional by means of the nozzle diameter $D$. Salinity concentration, horizontal and vertical velocity components are made non-dimensional using initial salinity $s_{j 0}$, cross-flow velocity $u_{c f}$ and initial jet velocity $u_{j 0}=\kappa u_{c f}$ (respectively).

\subsection{Semi-empirical models}

The plume dynamics is characterised by at least three phases:

- momentum phase: the plume is mainly driven by the initial momentum;

- buoyancy phase: the buoyancy force strongly pushes the plume upward/downward;

- entrainment phase: the cross-flow deviates the plume in horizontal direction and the counter-rotating vortex pair appears.

Such phases can occur simultaneously in a same spatial region. For example, if the buoyant force is weak with respect to the initial momentum the second and first phases overlap; hence a pure buoyancy driven phase does not exist even if the plume undergoes to the effects of buoyancy force.

Fischer et al. [17] derived two parameters that estimate the height at which the momentum and the buoyancy force strongly exercise their influence on the plume. The vertical length scale for the influence of initial momentum is

$$
z_{M}=\frac{\sqrt{Q_{j 0} u_{j 0}}}{u_{c f}}=\sqrt{\frac{\pi}{4}} D \kappa,
$$

while the scale for initial buoyancy force is

$$
z_{B}=\frac{B_{j 0}}{u_{c f}^{3}}=\frac{\pi}{4} \frac{D \kappa^{3}}{F^{2}},
$$

where the initial volume flux and the initial buoyancy flux are, respectively:

$$
Q_{j 0}=\frac{\pi}{4} D^{2} u_{j 0}, \quad B_{j 0}=\beta_{s} \Delta s Q_{j 0} g
$$

When $z_{B}>z_{M}$ the transition to the entrainment region starts after that $z^{*} \geq z_{B}$, otherwise when $z_{B}<z_{M}$ the transition occurs at

$$
z^{*} \geq z_{M}\left(\frac{z_{M}}{z_{B}}\right)^{1 / 3} .
$$

Empirical equations for the entrainment region have been given by Lee and Chu [30], under the main assumptions that in such region the plume moves horizontally 
with the cross-flow velocity and that the spreading rate of the solution is constant. The semi-empirical solution of the BJCF for the plume trajectory $\left(x_{j}, z_{j}\right)$ is:

$$
\begin{aligned}
z_{j} & =\left(\frac{3 B_{j 0} x_{j}^{2}}{2\left(1+k_{n}\right) \pi \beta^{2} u_{c f}^{3}}\right)^{1 / 3} \\
& =\left(\frac{3}{8\left(1+k_{n}\right) \beta^{2}}\right)^{1 / 3} \frac{D \kappa}{F^{2 / 3}}\left(\frac{x_{j}}{D}\right)^{2 / 3}
\end{aligned}
$$

where $x_{j}$ and $z_{j}$ are the stream-wise and vertical coordinate (respectively) with the origin placed where the entrainment region starts. The vertical and horizontal velocity components at the trajectory read, respectively:

$$
\begin{aligned}
w_{j} & =\left(\frac{4 B_{j 0}}{9\left(1+k_{n}\right) \pi \beta^{2} x_{j}}\right)^{1 / 3} \\
& =\left(\frac{1}{9\left(1+k_{n}\right) \beta^{2}}\right)^{1 / 3} \frac{u_{c f}^{2 / 3} \kappa^{2 / 3}}{F^{1 / 3}}\left(\frac{D}{x_{j}}\right)^{1 / 3}, \\
u_{j} & =u_{c f} .
\end{aligned}
$$

The empirical constants are estimated from experiment to be $k_{n}=1$ (mass coefficient) and $\beta \in(0.34,0.62)$ (calibration parameter).

The local BJCF radius $r_{j}$ and the salinity concentration of the plume with respect to the cross-flow $s_{j} / s_{j}$, have the following expressions:

$$
\begin{aligned}
r_{j} & =\beta z_{j}, \\
s_{j} / s_{j 0} & =\frac{Q_{j 0}}{\pi r_{j}^{2} u_{c f}}=\left(\frac{1+k_{n}}{3 \beta}\right)^{2 / 3} \frac{F^{4 / 3}}{\kappa}\left(\frac{D}{x_{j}}\right)^{4 / 3} .
\end{aligned}
$$

From these equations, the asymptotic behaviour of the BJCF plume in the bentover region is derived:

$$
\begin{aligned}
z_{j} \sim x_{j}^{2 / 3}, & w_{j} \sim x_{j}^{-1 / 3}, \\
r_{j} \sim z_{j}, & s_{j} / s_{j 0} \sim x_{j}^{-4 / 3} .
\end{aligned}
$$

\section{Simulation methodology and settings}

The fluid is considered incompressible and the Boussinesq approximation accounts for the buoyancy force generated by the salinity of the plume. The governing equations read:

$$
\begin{aligned}
\frac{\partial u_{i}}{\partial x_{i}} & =0 \\
\frac{\partial u_{i}}{\partial t}+u_{k} \frac{\partial u_{i}}{\partial x_{k}} & =-\frac{1}{\rho_{0}} \frac{\partial p}{\partial x_{i}}+\nu \frac{\partial^{2} u_{i}}{\partial x_{k} \partial x_{k}}-\frac{\rho}{\rho_{0}} g \delta_{i 3}, \\
\frac{\rho}{\rho_{0}} & =1-\beta_{s}\left(s_{j}-s_{0}\right)
\end{aligned}
$$

where $\delta_{i 3}$ is the Kronecker delta non-zero for the vertical component. 
The active scalar $s$ (salinity) is transported and diffused by the flow:

$$
\frac{\partial s}{\partial t}+u_{i} \frac{\partial s}{\partial x_{i}}=\alpha_{s} \frac{\partial^{2} s}{\partial x_{i} \partial x_{i}}
$$

where $\alpha_{s}$ is the molecular salinity diffusivity.

\subsection{LES approach and turbulence model}

The LES approach is adopted to model the turbulent content of the fluid flow (among the others Sagaut [39] and Piomelli [37]). The computational grid acts as an implicit spatial filter on the Navier-Stokes equation (18), and generates an extra term: the sub-grid scale (SGS) stress tensor $\tau_{i k}=\bar{u}_{i} u_{k}-\bar{u}_{i} \bar{u}_{k}$. The over-bar denotes the spatial filter that has width equal to local cell width, computed as $\bar{\Delta}=\left(\bar{\Delta}_{x} \bar{\Delta}_{y} \bar{\Delta}_{z}\right)^{1 / 3}$. The system is closed using the eddy viscosity assumption and the Smagorinsky model to determine the SGS viscosity $\nu_{\mathrm{SGS}}$, respectively:

$$
\begin{aligned}
\tau_{i k}-\frac{\delta_{i k}}{3} \tau_{\ell \ell} & =-2 \nu_{\mathrm{SGS}} \overline{S_{i k}} \\
\nu_{\mathrm{SGS}} & =c_{s}^{2} \bar{\Delta}^{2}\left|\overline{S_{i k}}\right|
\end{aligned}
$$

where $\overline{S_{i k}}=(1 / 2)\left[\left(\partial \bar{u}_{i} / \partial x_{k}\right)+\left(\partial \bar{u}_{k} / \partial x_{i}\right)\right]$ is the resolved strain rate tensor and $c_{s}$ is the Smagorinsky constant.

The dynamic model first developed in Germano et al. [22] allows computation of the constant of the model dynamically, based on the properties of the turbulent field. When the flow does not exhibit directions of homogeneity, the Lagrangian dynamic model of Meneveau et al. [34] offers a physical based framework to calculate the model constant. This SGS model has been implemented within the OpenFOAM platform and successfully used in inhomogeneous turbulence by Cintolesi et al. [6].

According to the dynamic procedure, an additional filtering operation of width $\widehat{\Delta}=2 \bar{\Delta}$ is applied to the resolved velocity field and to get information from larger scales of motion for computing the SGS viscosity. An average over a fluid particle trajectory is performed, thus making the model free from homogeneity over some spatial directions. The Smagorinsky constant is determined as:

$$
c_{s}^{2}=\frac{\mathcal{I}_{L M}(\mathbf{x}, t)}{\mathcal{I}_{M M}(\mathbf{x}, t)}
$$

The numerator and the denominator are given by a sequence specified by recursion on index $n$, respectively:

$$
\left\{\begin{array}{l}
\mathcal{I}_{L M}^{n+1}(\mathbf{x})=\epsilon\left[L_{i k} M_{i k}\right]^{n+1}(\mathbf{x})+(1-\epsilon) \cdot \mathcal{I}_{L M}^{n}\left(\mathbf{x}-\overline{\mathbf{u}}^{n} \Delta t\right) \\
\mathcal{I}_{L M}^{0}(\mathbf{x})=c_{s, 0}\left[M_{i k} M_{i k}\right]^{0}(\mathbf{x})
\end{array}\right.
$$

and

$$
\left\{\begin{array}{l}
\mathcal{I}_{M M}^{n+1}(\mathbf{x})=\epsilon\left[M_{i k} M_{i k}\right]^{n+1}(\mathbf{x})+(1-\epsilon) \cdot \mathcal{I}_{M M}^{n}\left(\mathbf{x}-\overline{\mathbf{u}}^{n} \Delta t\right) \\
\mathcal{I}_{M M}^{0}(\mathbf{x})=\left[M_{i k} M_{i k}\right]^{0}(\mathbf{x})
\end{array}\right.
$$




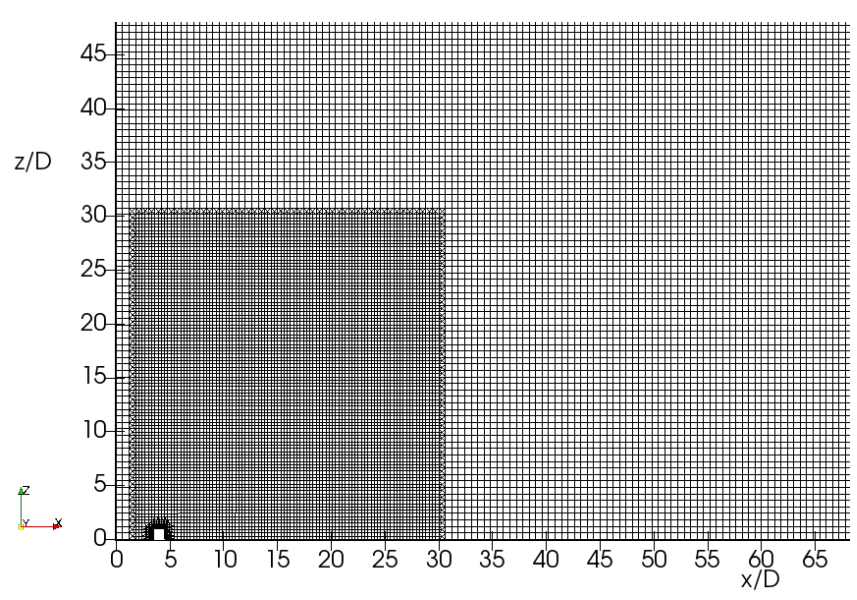

Fig. 2: Computational mesh: refinement region in the neighbourhood of the nozzle.

where the index 0 denotes the quantity calculated at the first iteration, $c_{s, 0}=$ 0.0256 is the standard Smagorisky constant, $\Delta t$ is the simulation time step, and

$$
\epsilon=\frac{\Delta t / \mathrm{T}^{n}}{1+\Delta t / \mathrm{T}^{n}}, \quad \mathrm{~T}^{n}=1.5 \bar{\Delta}\left(\mathcal{I}_{L M}^{n} \mathcal{I}_{M M}^{n}\right)^{-1 / 8},
$$

while

$$
L_{i k}=\widehat{\bar{u}}_{i} \bar{u}_{k}-\widehat{\bar{u}}_{i} \widehat{\bar{u}}_{k}, \quad M_{i k}=2 \bar{\Delta}^{2}\left(\left|{\widehat{\bar{S}} \mid \bar{S}_{i k}}-4\right| \widehat{\bar{S}} \mid \widehat{\bar{S}}_{i k}\right)
$$

Since the $c_{s}^{2}$ cannot assume negative values, a numerical clipping is done on $\mathcal{I}_{L M}$, $\mathcal{I}_{M M}$ in order to be always positive.

Similarly, the filtering operation applied to the transport equation of the scalar concentration (20) generates a salinity SGS flux $\lambda_{i}=\overline{s u}_{i}-\bar{s} \bar{u}_{i}$. Such an extra term is modelled in a similar manner as for the SGS stress tensor, applying a dynamic Lagrangian procedure to close the equation (see Armenio and Sarkar [1]). For the sake of brevity, the equations for active scalar are not here reported; a complete description of the model used can be found in Cintolesi et al. [7, 8].

\subsection{Computational mesh}

To the best of the authors' knowledge there are no criteria for grid spacing in the BJCF case; however, de Wit and van Rhee [10] suggested to use a mesh with a cell size around $0.1 D$. A computational grid with such cell width would lead to very high computational cost in the present case. Hence, we use a fine mesh around the jet exit (cells size $0.2 D$ or smaller) and a coarser mesh elsewhere (cell size around $0.5 D)$. This choice is reasonable since the turbulent structures are smaller above the nozzle (when plume velocity magnitude is high) while they become larger when the plume penetrates the cross-flow.

The computational grid has been created using the snappyHexMesh tool of OpenFOAM 2.3.0. The background mesh is composed by a uniform grid that 
Table 1: Physical parameters and boundary conditions.

\begin{tabular}{l|l}
\hline Fluid parameters & Flow conditions \\
\hline$\nu=1 \times 10^{-6}$ & $u_{j 0}=8.2 \times 10^{-1} \mathrm{~m} / \mathrm{s}$ \\
$\beta_{s}=7.5 \times 10^{-4}$ & $u_{c f}=1 \times 10^{-1} \mathrm{~m} / \mathrm{s}$ \\
$\alpha_{s}=1.43 \times 10^{-9}$ & $\Delta s=9.1 \times 10^{1}$ \\
\hline
\end{tabular}

discretises the computational domain (see section 2.1) in $200 \times 66 \times 116$ points in the $x, y, z$ directions (respectively). The mesh is refined (width of cells is divided by a factor 2 in each direction) in a zone around the nozzle, consisting of a box of dimensions $28 D, 6 D, 30 D$ shown in Figure 2. Such a box has one face on the bottom of the computational domain, is centred with respect to the $y$-direction, and is placed at a distance of $x / D=2$ from the inflow face. Above the nozzle, the momentum and buoyancy phases occur and also the entrainment starts. A thin region around the cylinder is additionally refined for better capturing the nozzle shape. The final mesh has 1912023 points.

\subsection{Parameters setting, initial and boundary conditions}

The experimental case reproduced is the Run 10-8 reported by Fan [16]. The case is characterised by Froude number $F=10$, velocity ratio $\kappa=8$, and Reynolds number $R e=8200$.

Table 1 reports the fluid physical parameters and the flow boundary conditions employed in accordance with the experimental settings. The salinity diffusion coefficient is estimated as $\alpha_{s}=\nu / S c$, where the Schmidt number is $S c=0.7 \times 10^{3}$. The plume is composed by salt water while the ambient fluid is fresh water, thus $s_{c f}=0$ and $s_{j 0}=\Delta s=91$. Regarding the use of the Boussinesq approximation, Gray and Giorgini [23] suggests that it is valid up to a variation of $10 \%$ of fluid thermophysical properties. Employing the above-reported settings, density variation is estimated to be $\Delta \rho / \rho_{0}=\beta_{s} \Delta s \sim 7 \%$; hence, simulation is within the range of validity of the Boussinesq approximation. Because of visualisation reasons, the gravity force is changed of sign and, thus, the plume is pushed upward instead of downward.

The boundary conditions are: inlet, constant velocity $u_{c f}$ in $x$-direction and salinity $s_{c f}=0$; lateral and top boundaries, constant velocity $u_{c f}$ in $x$-direction and zero-gradient for salinity; bottom boundary and nozzle cylinder are considered solid surfaces, hence no-slip condition for velocity and zero-gradient for salinity; nozzle exit, constant velocity $u_{j 0}$ in $z$-direction and salinity $s_{j 0}=91$; outlet, zerogradient for velocity and salinity. Pressure is fixed as $p=0$ at the outlet, while the zero-gradient condition is set elsewhere. The Spalding wall-function is used at the solid boundaries, see Launder and Spalding [28].

Notice that in the reference experiment the bottom boundary was the ambient water free surface. Because of some computational difficulties that can arise when the solid nozzle interacts with a free surface, the authors decided to simulate a solid surface instead. This choice does not alter the simulation since the boundary layer arising at the solid surface does not influence the plume dynamics, that develops sufficiently far from such boundary. 
To stabilise the computation and to facilitate the outflow of the plume, a sponge region with a decreasing buoyancy force is generated in the last part of the domain in stream-wise direction (see Armenio [2]): at $x / D=85$ the gravity acceleration starts to decrease linearly till $x / D=115$ when it is set to zero.

In order to speed up the simulation, the configuration used to initialised the case is a steady-state solution, obtained by a RANS simulation with a $k-\epsilon$ turbulent model.

\subsection{Algorithm and numerical schemes}

The open-source software OpenFOAM version 2.3.0 is employed for the simulation. The basic solver buoyantBoussinesqPimpleFoam has been customised to perform the above described LES: a body force term was added to drive the flow and the numerical clipping on salinity. Moreover, the dynamic Lagrangian sub-grid scale model previously described has been implemented and used for the simulation; such a model has been originally developed within the IEFLUDS research group of the University of Trieste. The resolution algorithm is PISO (see Oliveira and Issa [36]); more information about the basic solver can be found in the OpenFOAM documentation.

The governing equations are discretised in time using an implicit Euler backward scheme (that uses the variables at the previous two time steps, resulting in second order accuracy); in space using a second-order central difference scheme for all terms except the convective ones in momentum and salinity equations. The discretisation of such terms is a critical issue in LES, since they can lead to numerical instabilities (wiggles) in the region where the plume acts as an obstacle for the cross-flow and sharp interface develops. The salinity advection term is discretised with the MUSCL scheme by van Leer [41] as it is implemented in OpenFOAM, while for momentum advection we used the Gauss-Gamma scheme proposed by Jasak et al. [26] with $\gamma=0.2$. The former is a total variation diminishing scheme of second-order accuracy, the latter is a bounded version of central differencing for unstructured meshes based on the normalised variable diagram. A numerical clipping on salinity is performed to prevent non-physical values. A simulation without clipping shows that such non-physical values appear in a few cells near the bottom wall and their magnitude remains limited in time. Elsewhere, such a simulation does not exhibit detectable discrepancies with respect to the actual one.

\section{Validation}

The validation of simulation results is done against experimental data of Fan [16] and the semi-empirical relations reported in section 2.3.

After that a statistical steady state was established, the simulation ran for a period equal to $3 t_{0}$, where $t_{0}=h / u_{c f}=12 \mathrm{~s}$ is the time required by the cross-flow to pass through the entire domain. The statistics are collected over this period. The averaging operation is notated with angular bracket $\langle\psi\rangle$. The system is symmetric with respect to the vertical centre plane $y / D=0$. 


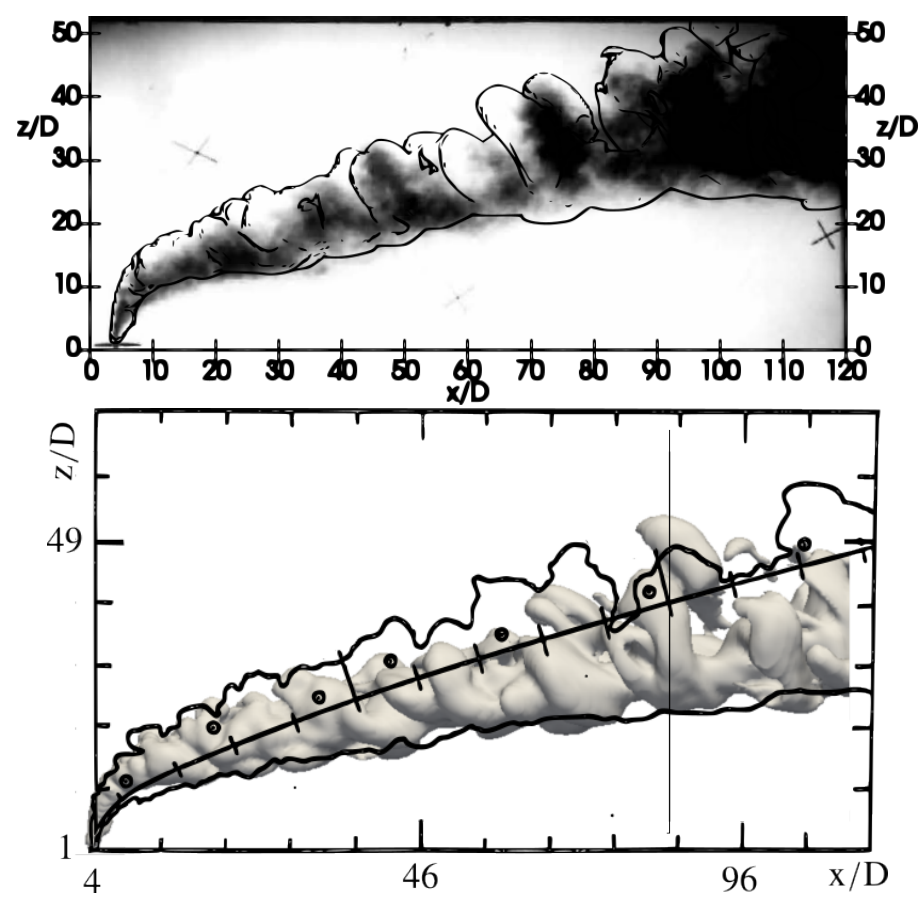

Fig. 3: Qualitative comparison between experiment [16] and LES plume (visualised by $s_{j}=0.01 s_{j 0}$ isosurface). Top: overlap of experimental plume photograph with simulated plume profile. Bottom: overlap of experimental plume profile with simulated plume. The vertical solid line delimits the buoyancy-free region.

4.1 Instantaneous plume comparison

The instantaneous plume at time $2 t_{0}$ is reported together with the experimental pictures for a qualitative comparison. The LES plume is visualised by $s_{j}=0.01 s_{j 0}$ isosurface, while the experimental plume was marked by black tracing ink and quantitative measures on the value of the scalar at the interface are not available. In Figure 3 the simulated plume profile overlaps to experimental plume photograph (top figure), and the experimental plume profile overlaps to the LES plume salinity isosurface (bottom figure). Overall, we can appreciate a good qualitative agreement between the simulated plume shape with the experimental one. In bottom figure, along stream-wise direction the simulated plume maintains a lower height and slightly deflects downward. This is expected because of the presence of the buoyancy-free region (delimited by the vertical black line in the figure). Even though it is just a qualitative comparison, it shows the LES capability to reproduce trustworthy the instantaneous flow features. A detailed analysis of such features is proposed in the following sections. 


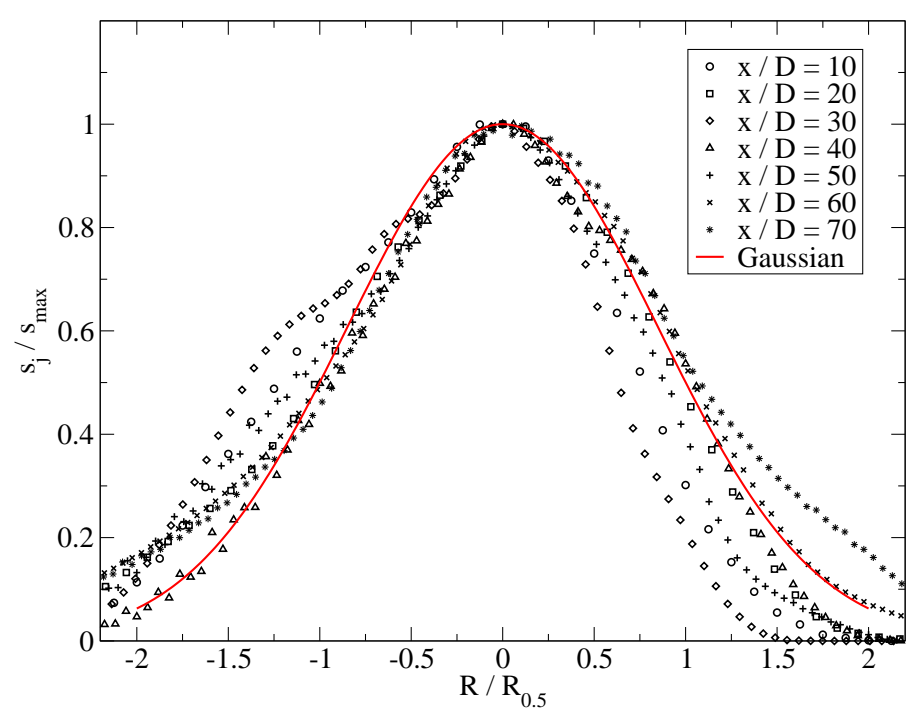

Fig. 4: Non-dimensional concentration versus non-dimensional plume radius. The symbols are the LES data taken at the stream-wise position $x / D$. The red line is the Gaussian error function that fits the experimental data of Fan [16].

\subsection{The mean field}

The LES results are validated against the available experimental data and semiempirical relations. Fan [16] provides experimental data about salt concentration distribution and the plume half-width. The concentration profile is taken along a vertical centre-line (with respect to spanwise direction), where it exhibits a Gaussian distribution. The point where the concentration reaches the maximum $s_{\max }$ is taken as the centre of the plume section. The half-width radius $R_{0.5}$ is then defined as the distance between the centre and the point where $s_{j}=0.5 s_{\max }$.

Figure 4 shows the normalised concentration distribution with respect to the radial coordinate $R$ (the distance with sign from the plume centre) normalised by the half-width radius. The averaged concentration profiles at different stream-wise levels $x / D=10,20,30,40,50,60,70$ are reported, along with the Gaussian error function that fits experimental data: $G(\xi)=e^{-\xi^{2} / 2 \sigma}$, where $\sigma=0.85$. Overall, the simulation profiles follow the Gaussian distribution with some skewness due to the action of the cross-flow.

Figure 5 reports the non-dimensional half-width radius, together with the theoretical relation proposed by Fan [16] and the quadratic regression curve for simulation data. Since there is a slight discrepancy between the half-width radius measured above and below the plume centre, $R_{0.5}$ is here computed as the average between the two. LES results are generally well correlated with the experimental data; a much better agreement is not expected because the latter are measured at an instantaneous plume and they are affected by a measurement error of $2-10 \%$. Notice that both experimental and LES data do not follow the theoretical relation 


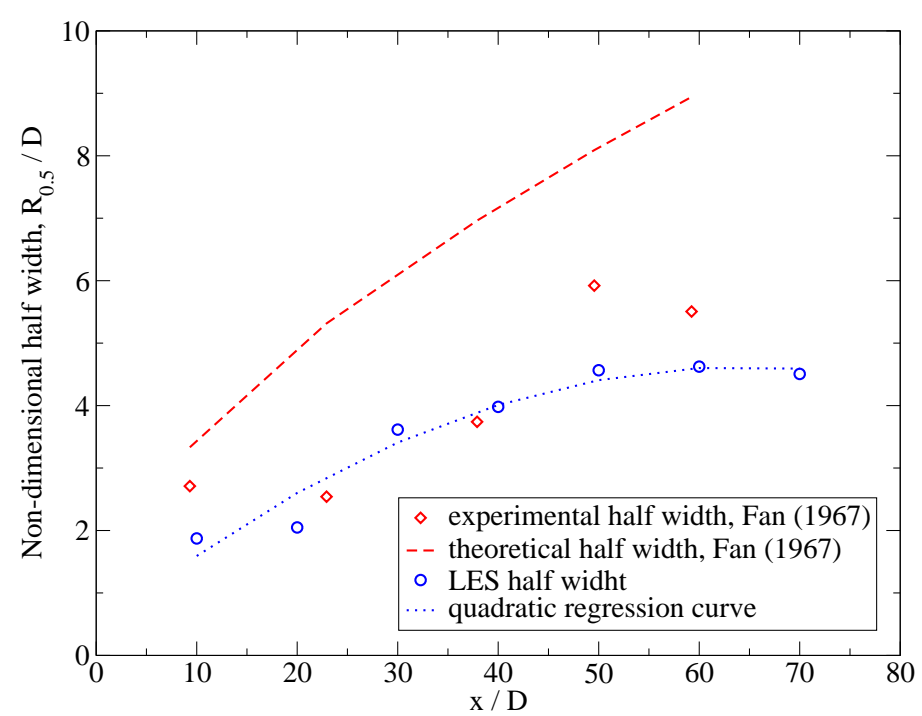

Fig. 5: Non-dimensional half-width at different stream-wise levels. Dashed line: theoretical relation proposed by Fan [16]; Dotted line: quadratic regression curves for LES data.

proposed by Fan [16], that fits better the data of the cases at higher Froude numbers. The simulation suggests a polynomial increment of the half-width radius.

In order to compare the results with semi-empirical relations reported in section section 2.3, the problem-dependent constants $\beta$ and $k_{n}$ in equations (9),(11) and (13) have to be determined. The latter is set $k_{n}=1$ as recommended in literature. The height of the plume trajectory $z_{j}$ and the radius $r_{j}$ of the plume at a vertical plane orthogonal to the cross-flow are measured and used to determined $\beta$, see equation (13).

The plume trajectory is defined as the streamline starting from the centre of the nozzle, and developing in the vertical centre-plane parallel to the cross-flow. Following Lee and Chu [30] the radius is computed as:

$$
r_{j}=\sqrt{R_{h} R_{v}}
$$

where $2 R_{h}$ and $2 R_{v}$ are horizontal and vertical (respectively) distance between the two far ends of the $0.01 s_{j 0}$ level-set at vertical plane $x / D=$ costant. Note that in [30] the suggested value for isosurface is $0.25 s_{j 0}$. The one-per-cent salinity isosurface is chosen because it labels approximately the $99 \%$ of total plume material, while the twenty-five-per-cent contour takes into account just the $86 \%$ of material (approximately). In the present case, the latter does not allow a complete visualisation of the plume because of the fast dilution rate of salt. It is worth to mention that the estimation formula (28) is not the only one proposed in literature. For example, de Wit et al. [11] found a good agreement with semi-empirical formula when $r_{j}$ is computed as the radius of a circle with an area similar to the region delimited by the $0.01 s_{j 0}$ level-set. In our case, such estimation of the radius gives values similar to the formula (28): the relative difference is around $2 \%$ till the point 


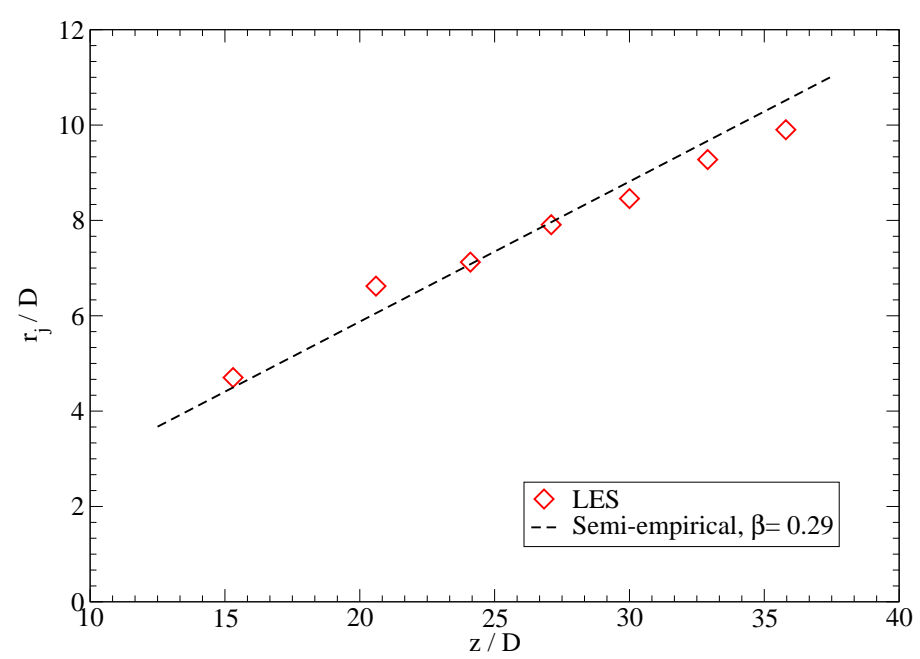

Fig. 6: Non-dimensional plume radius versus non-dimensional height of the plume trajectory. The data are taken at $x / D=10,20,30,40,50,60,70$, respectively from left to right.

$x / D=40$ and increases up to $10 \%$ at the point $x / D=70$, essentially because the one-per-cent level-set splits into two regions (see Figure 13). In general, there are ambiguities in the definition of the radius: it depends both on the level-set value adopted and on the evaluation formulae chosen. Such ambiguities can lead to different evaluations of the radius and, thus, to different estimations of $\beta$.

Figure 6 shows the non-dimensional plume radius versus the trajectory height. The radii are measured at planes $x / D=10,20,30,40,50,60,70$. The direct proportionality between the two quantities holds, and the simulated plume fits well the semi-empirical relation (13) when $\beta=0.29$ is chosen. Such value is slightly out the range reported in literature. This may be due to the different methods used for radius estimation.

Figure 7 reports the simulated centre-line trajectory together with experimental data and semi-empirical relation (9) for three values of $\beta$. The semiempirical formulae are valid in the entrainment region, that starts approximately at $z^{*} / D=8.65$ and $x^{*} / D=1.24$; where the critical height $z^{*}$ is computed through equation (7). The LES trajectory is in a very good agreement with the experimental data and practically collapses to the semi-empirical profile (9) when $\beta=0.48$ is chosen. On the contrary, when $\beta=0.29$ is used the two plots differ. This issue is also reported in [11] where different values of $\beta$ were used for trajectory and salinity concentration. The trajectories computed using maximum and minimum recommended values of $\beta$ are also reported.

Figure 8 presents the salinity profiles. The salinity concentration $s_{j}$ is estimated in two ways, respectively as the maximum and the mean salinity values in vertical planes orthogonal to the cross-flow. In top panel, salinity is compared with equation (14), where $\beta=0.29$ is used because it is found to be more suitable for relations that involve the radius. The mean salinity behaviour is very well described by the semi-empirical relation. The maximum salinity follows a similar 


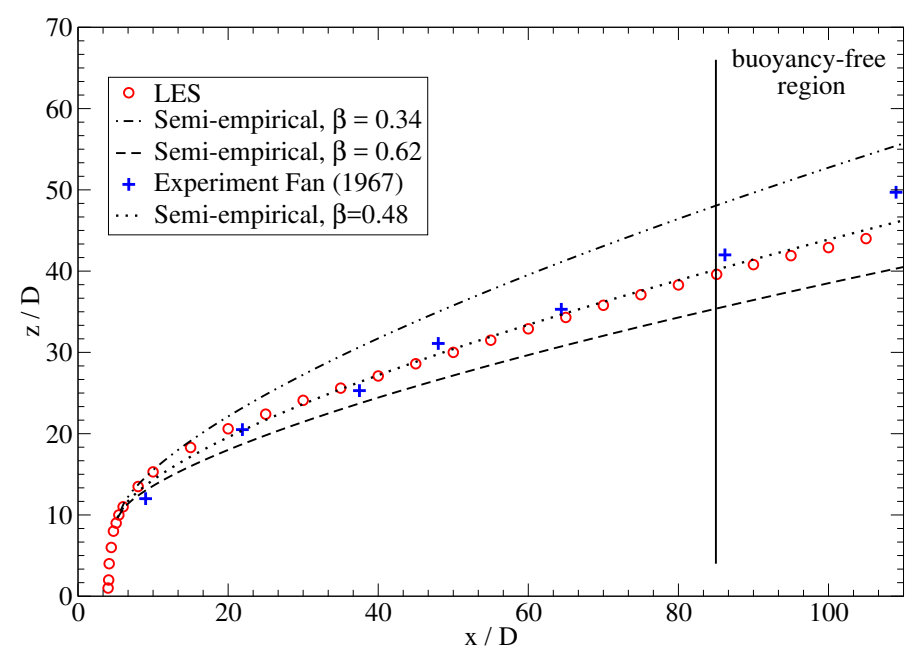

Fig. 7: Plume trajectory at the vertical centre plane. Comparison between the present numerical simulations (LES), the semi-empirical formulae for two values of $\beta$, and the experimental data of Fan [16].
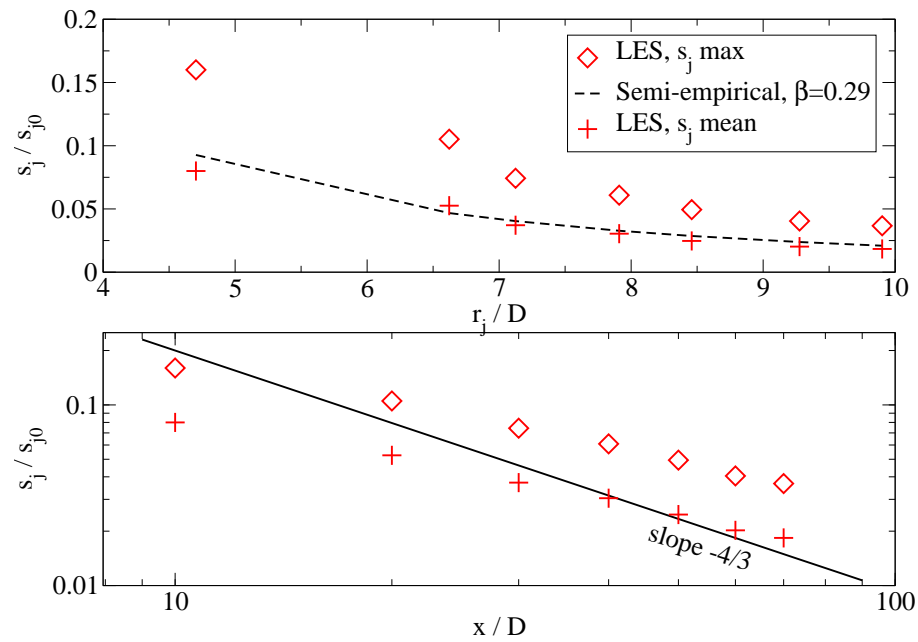

Fig. 8: Non-dimensional salinity concentration compared with semi-empirical relations. Top: salinity concentration versus plume radius (data at the same planes as in Figure 6). Bottom: salinity in stream-wise direction compared with the asymptotic behaviour. 


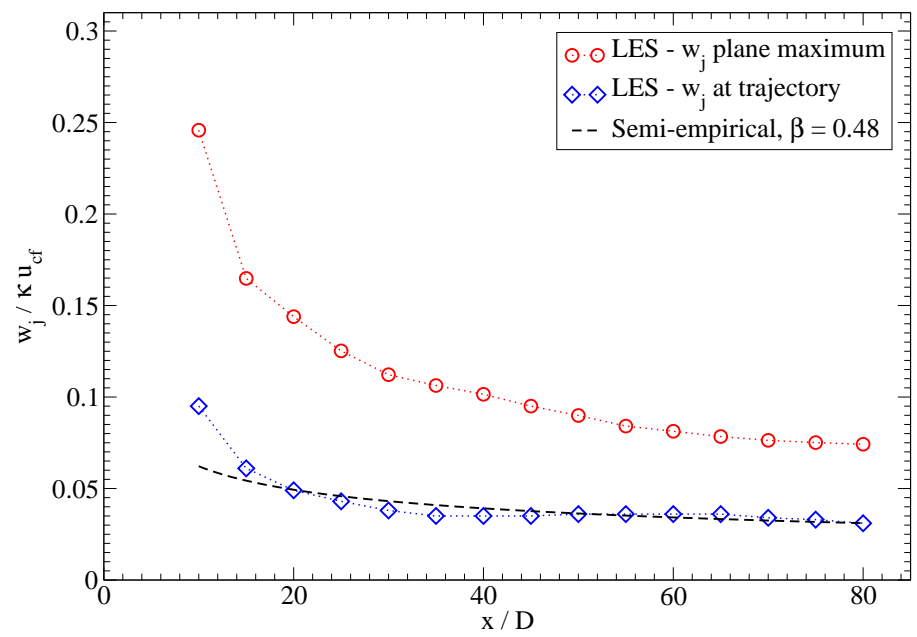

Fig. 9: Non-dimensional vertical velocity component of the plume versus nondimensional distance from the nozzle.

behaviour but exhibits higher values. In bottom panel, salinity spatial evolution is compared with the asymptotic trend (16) of the bent-over phase. The results do not agree with the reference, showing a different slope: simulated salinity decreases more gradually. However, it can be pointed out that the value of salinity can be estimated using other methods, which possibly allow a better agreement.

Figure 9 displays the non-dimensional vertical velocity component $w_{j}$ of the plume. It is evaluated in two ways: as the vertical velocity at the plume trajectory $z_{j}$, and as the maximum value of vertical velocity in a plane $x / D=$ cost. The former is in good agreement with the semi-empirical relation (11), when the value $\beta=0.48$ is used. Some discrepancies appear close to the nozzle where the influence of initial momentum and buoyancy force is still present. The latter overestimates the velocity and shows a slightly higher rate of decrease in stream-wise direction.

Overall, the simulation results are in excellent agreement with the reference data and formulae. Even if some uncertainties are pointed out in the calibration of parameter $\beta$, the profiles of the semi-empirical relations are well reproduced.

\subsection{Grid quality assessment}

To the best of authors knowledge, well-established criteria to build a suitable computational grid for BJCF is not present in literature. For the present grid, the following requirements in terms of grid spacing are adopted: above the nozzle (where the momentum-buoyancy phase drives the plume) maximum cell width is $0.2 D$; far from the nozzle (where the entrainment region is) cell width is around $0.5 D$. The quality of the grid is discussed hereafter. 


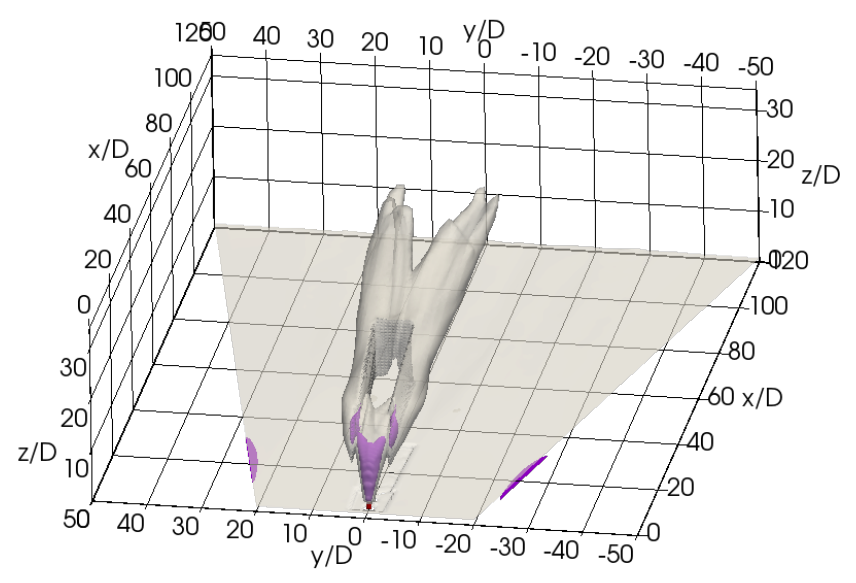

Fig. 10: Grey surface; comparison between the local cell width $\Delta$ and the Kolmogorov length-scale $\eta$ : isosurface at $\Delta / \eta=12$. Violet surface; comparison between the SGS and molecular dynamic viscosity: isosurface at $\nu /\left(\nu+\nu_{\mathrm{SGS}}\right)=0.33$. Transparency effect is used for a simultaneous visualisation.

First, the Kolmogorov length-scale $\eta$ is compared with the local cells size $\Delta$. The Kolmogorov scale is the characteristic length of dissipative motion, defined as

$$
\eta=\left(\frac{\nu^{3}}{\langle\epsilon\rangle}\right)^{1 / 4}
$$

where $\epsilon$ is the total dissipation rate of turbulent kinetic energy (TKE), computed as the sum of the resolved and SGS part:

$$
\epsilon=2 \nu\left\langle\mathrm{S}_{i k} \mathrm{~S}_{i k}\right\rangle+\epsilon_{\mathrm{SGS}}
$$

with $\mathrm{s}_{i k}=(1 / 2)\left[\left(\partial u_{i}^{\prime} / \partial x_{j}\right)+\left(\partial u_{j}^{\prime} / \partial x_{i}\right)\right]$ is the fluctuation strain rate tensor and $\epsilon_{\mathrm{SGS}}=\left\langle\tau_{i j} \overline{S_{i j}}\right\rangle$ is the SGS scales contribution (see Armenio and Sarkar [1] and Cintolesi et al. [8]).

In an anisotropic turbulence case, the maximum dissipation is due to lengthscale of the order of $24 \eta$ (see Pope [38]). At least two computational points are needed to capture the flow features; hence, a computational grid satisfying $\Delta / \eta \leq$ 12 characterised a well-resolved region (see Fröhlich et al. [19] and Cavar and Meyer [4]). Figure 10 reports on grey colour the isosurface of $\Delta / \eta=12$. It is clearly visible the refinement region where the ratio values decay, while in the momentumbuoyancy phase of the plume turbulence is more intense and characterised by smaller length-scale than in rest of the domain. Inside the region delimited by the isosurface, the ratio can assume higher values but remains limited to $\Delta / \eta<24$, except in few cells above the nozzle and at the top lateral boundaries of the refinement region (see the following discussion). The number of such cells is limited and they do not affect the overall fluid dynamics of the BJCF. The above discussion holds for a direct simulations; thus, the grid is fine enough for a detailed analysis of the flow field using LES. 


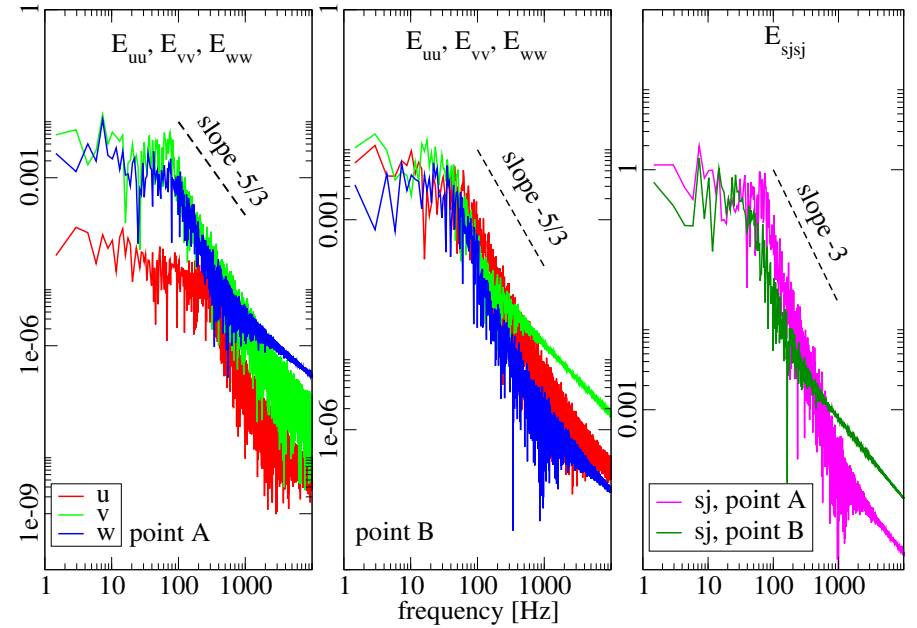

Fig. 11: Power spectra of the three velocity components $\left(E_{u u}, E_{v v}\right.$ and $\left.E_{w w}\right)$, and salinity $\left(E_{s j s j}\right)$ at the two points in the vertical centre plane parallel to the cross-flow: $A=(5 D, 8.64 D)$ and $B=(10 D, 15 D)$. Interval of sampling: $0.6 t_{0}$; data collected every $6.1 \times 10^{-6} t_{0}$.

Second, the turbulent and molecular viscosity can be compared to estimate the contribution of the SGS model. Figure 10 shows in violet colour the isosurface of $\gamma=\nu /\left(\nu+\nu_{\mathrm{SGS}}\right)=0.33$. Such quantity is limited by $\gamma \in[0,1]$ where a value close to one means that LES reduces to a quasi direct numerical simulation, while values close to zero imply a high contribution of turbulent model. The value $\gamma<0.5$ indicates a grid able to capture the large part of turbulence structures; $\gamma=1 / 3$ corresponds to a ratio between SGS and molecular viscosity of $\nu_{\mathrm{SGS}} / \nu=2$. The ratio is higher than two in a limited zone above the nozzle and at the top-lateral boundaries of the refinement region. The latter is due to the fact that in the last part of the momentum-buoyancy phases, the plume is slightly larger than the refinement region. At the edge of such region the grid is coarser but the plume still triggers some turbulence. Above the nozzle, turbulence is more intense and thus the contribution of the SGS model increases but the value $\gamma=0.25$ is not exceeded.

Third, the time power spectra of velocity components and salinity are analysed (see de Wit et al. [11] and Cavar and Meyer [4]). They are computed by using the Fourier transform of data collected at two probes in the vertical centre plane, located at points: $A=(5 D, 8.64 D)$ at the boundary between momentum-buoyancy and entrainment phases; $B=(10 D, 15 D)$ at the plume trajectory. Velocity spectra decay with a slope of approximately $-5 / 3$, while the salinity spectra follow a slope of -3 .

Overall, the above-reported analyses confirm that the computational grid used is suitable for a reliable LES. A finer computational grid can be used in nozzle zone in order to reduce the contribution of the SGS model where the plume turbulence is more intense: a grid width of $0.1 D$ (the half of the actual width) is suggested because it satisfies the constrain $\Delta / \eta<12$ and, hence, it leads to a well resolved 


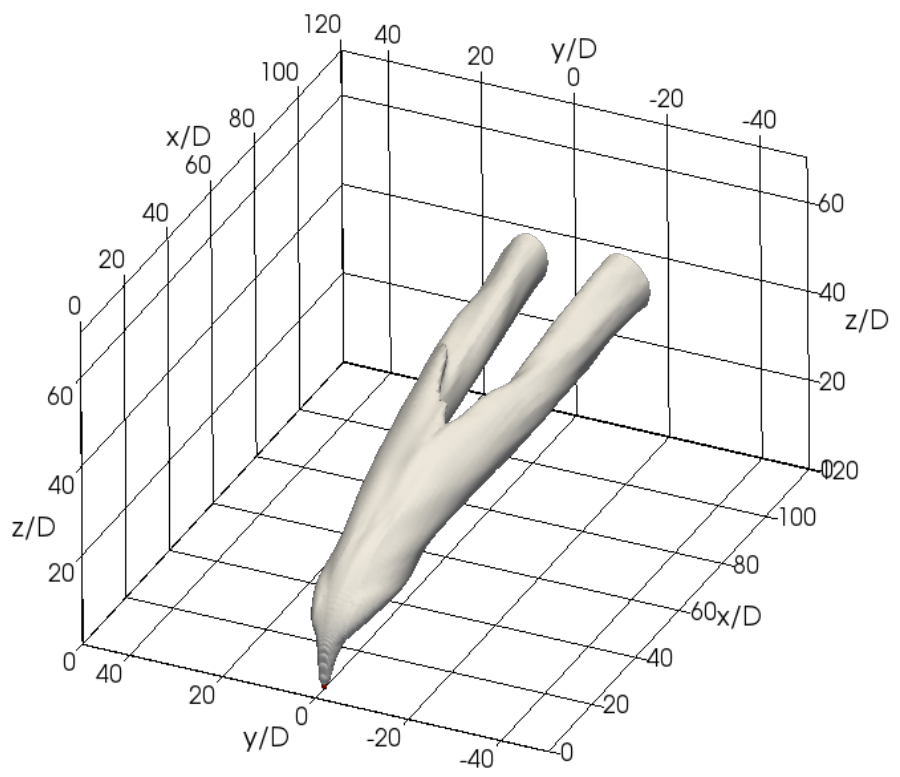

Fig. 12: Contour-plot of average salinity $\left\langle s_{j} / s_{j 0}\right\rangle=0.01$.

LES. However, a coarser grid in such region does not affect the general accuracy of the results, as proven in validation section section 4.2 .

\section{Mean flow analyses}

The first order statistics are here reported. The different phases of the plume dynamics can be analysed in light of the characteristic vertical length scales, see equations (4),(5) and (7):

$$
z_{M} / D=7.3, \quad z_{B} / D=4.3, \quad z^{*} / D=8.65 .
$$

In the flow under analysis $z_{M}>z_{B}$, thus a pure buoyancy phase is not present: buoyancy force affects the plume at a short distance above the nozzle. After the height $z^{*}$ the cross-flow overcomes initial momentum and the entrainment governs the plume dynamics.

Figure 12 reports the average one-per-cent salinity isosurface. The plume shows a good symmetry with respect to the vertical centre plane $y / D=0$. Above the nozzle (approximately $z<z^{*}$ ) the plume has a cylindrical shape, whereas farther it starts to enlarge in spanwise direction. After $z>z^{*}$ two typical counter-rotating vortex pair (CVP) become clearly visible. Such vortices generate two branches that are linked at the top by a layer of salt water, that becomes thinner and thinner as the plume is transported by the cross-flow. The two vortices separate approximately at $x / D=58$ and assume a regular cylindrical shape.

Figure 13 allows for a more precise description. It displays the average velocity components and salinity level-sets at four selected vertical planes at $x / D=$ 

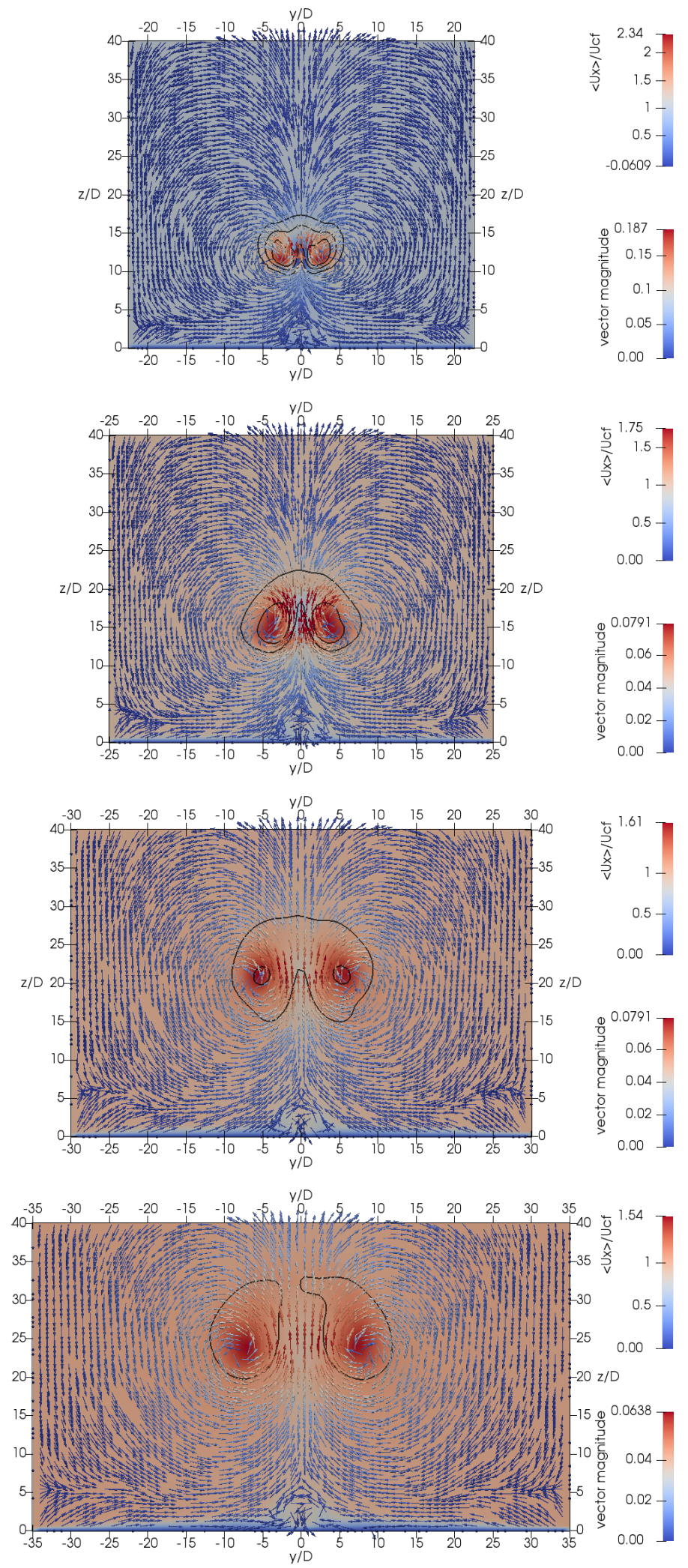

Fig. 13: Plane distribution of non-dimensional stream-wise velocity $\left\langle u_{x} / u_{c f}\right\rangle$, together with salinity contour-line at levels $\left\langle s_{j} / s_{j 0}\right\rangle=0.01,0.05,0.1$ and the planar velocity vectors $\left(\left\langle u_{y}\right\rangle,\left\langle u_{z}\right\rangle\right)$. Vertical planes at $x / D=10,20,40,60$ from the top to the bottom, respectively. 


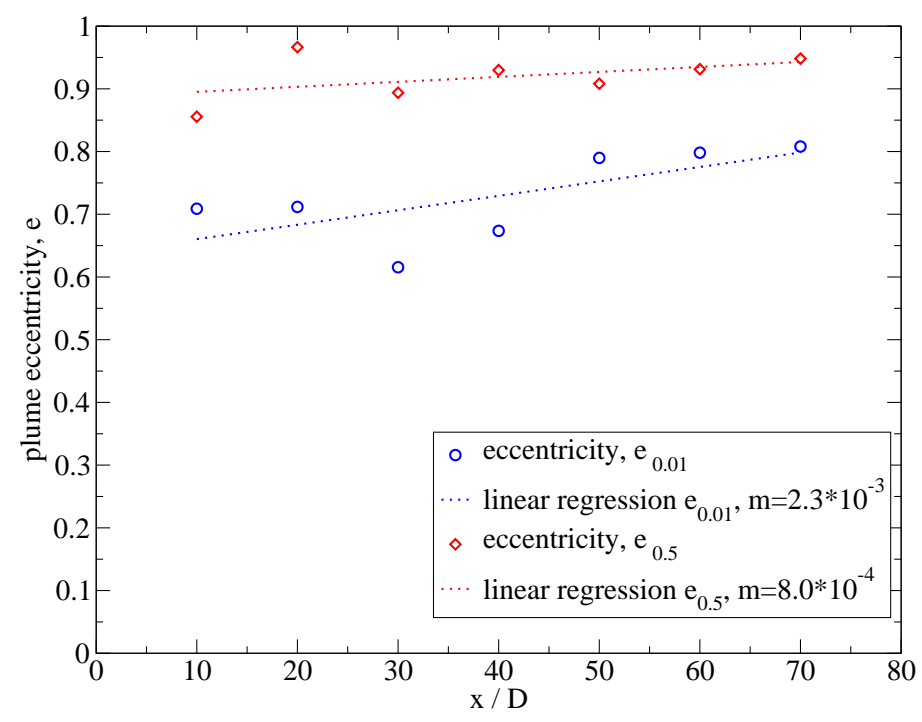

Fig. 14: Eccentricity of the plume vertical section along stream-wise direction: $e_{0.01}$ is computed using the plume radius; $e_{0.5}$ is computed using the half-width radius. The regression lines of the two data sets are also reported, together with the respective slope $m$.

10, 20, 40, 60 along stream-wise direction. The non-dimensional stream-wise velocity component is reported as colour distribution, while the vertical and spanwise velocity components are displayed by means of vector arrows coloured by vector magnitude $\left(u_{y}^{2}+u_{z}^{2}\right)^{1 / 2}$. In all the planes, the local maximum of $u_{x}$ is approximately coincident with the salinity local maximum. It is expected since salt is mainly transported than diffused. In between the two vortices, the stream-wise velocity has a minimum. The vectors show the counter-rotating flow around these points. It can be noticed that vertical velocity is more intense between and just under the vortex pair, while it is almost negligible elsewhere. Salinity level-set has the typical kidneys shape: the vertical velocity (triggered by the CVP) injects fresh water from the bottom, giving rise to a sort of groove in the plume surface along the stream-wise direction. Salinity diffuses more in the higher part because of the turbulent interaction with the cross-flow. Indeed, the plume top boundary is more affected by mixing turbulent structures (see section 6).

At level $x / D=10$, the flow is more energetic (all velocity components reach higher values) and the plume is still concentrated in a limited region. The velocity component $u_{x}$ is very small in the region between the vortices (see also Figure 15). In fact, the plume above the nozzle acts as an obstacle to the cross-flow and generates a sort of stagnant region of almost zero stream-wise velocity. At level $x / D=20$ the flow is less intense and $u_{x}$ reduces. The salinity diffuses in the spanwise direction, and the level-set $\left\langle s_{j} / s_{j 0}\right\rangle=0.1$ is not anymore visible. At levels $x / D=40,60$ the stream-wise velocity smoothly decreases, while the magnitude of other velocity components essentially maintains low values. The separation of the plume branches is visible in $x / D=60$ plane . 


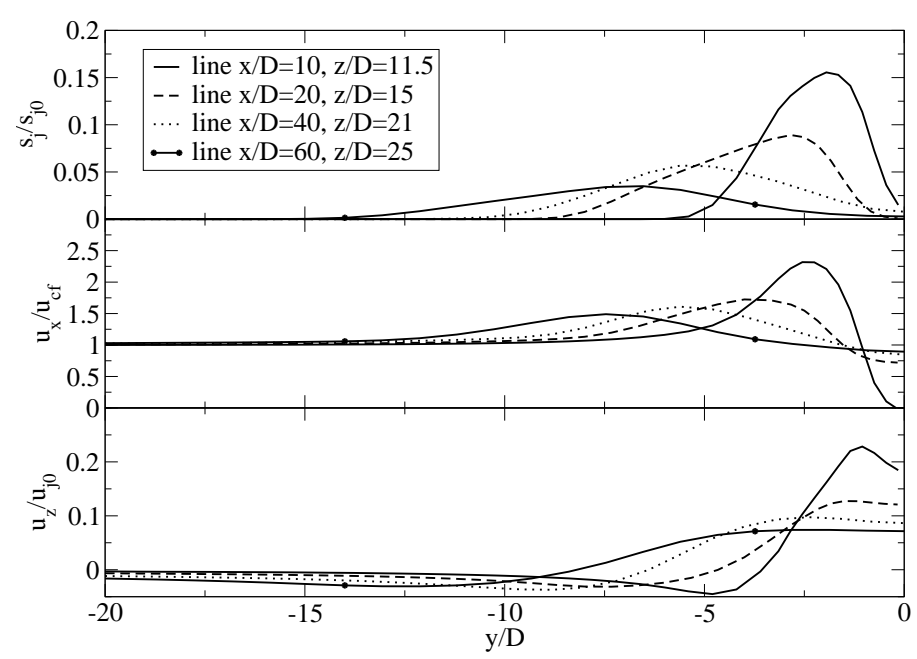

Fig. 15: Profiles of non-dimensional mean salinity concentration, stream-wise and vertical velocity at four selected line in spanwise direction: $(x / D, z / D)=$ $(10,11.5) ;(20,15) ;(40,21) ;(60,25)$. The quantities are averaged in time and space, taking advantage of the symmetry plane $y / D=0$.

Figure 14 shows the plume eccentricity on a vertical cross-section. It is defined as $e=\left(1-R_{v} / R_{h}\right)^{1 / 2}$, where $R_{v}$ and $R_{h}$ are the vertical and horizontal radii, respectively. Two estimation techniques of such radii lead to two evaluations of eccentricity (see also section 4.2 for radius estimation techniques): for $e_{0.01}$ the radii are the distance between the two far ends of the one-per-cent level set of salinity (in vertical and horizontal directions); for $e_{0.5}$ the radii are computed as the maximum distance between the plume centre and the level set $0.5 s_{\max }$ (in horizontal and vertical directions), i.e. the half-width radius definition proposed by Fan [16] is employed. Both quantities exhibit values higher than 0.5 and weak linear growth in stream-wise direction: the slope $m$ of the regression line is close to zero. Hence, the plume has a strong elliptic shape that increases in extension but does not vary considerably its eccentricity. The quantity $e_{0.5}$ is related to the plume core and exhibits higher values remaining almost constant as the plume develops. The quantity $e_{0.01}$ takes into account a larger plume boundary, it has lower values and a slightly higher increase along the stream-wise direction.

Figure 15 reports the non-dimensional profiles of salinity, $u_{x}$ and $u_{z}$ velocity components along selected lines in $y$-direction. The average is also done in space using the symmetry of the system with respect to $y / D=0$. Hence, just the profiles for half line $y / D<0$ are shown. The four lines belong to the same vertical planes as in Figure 13 and are chosen in such a way to pass by (approximately) the point of local maximum of stream-wise velocity. The salinity profiles clearly show the salinity decays between the vortex pair at all the planes. As long as the plume is entrained, the overall salt concentration decreases and the local peak is displaced far from the symmetry plane. The stream-wise velocity profiles also exhibit similar features: moving downstream the local peak moves laterally from centre line and assumes values around $\% 50$ higher than the cross-flow velocity. It is worth to 


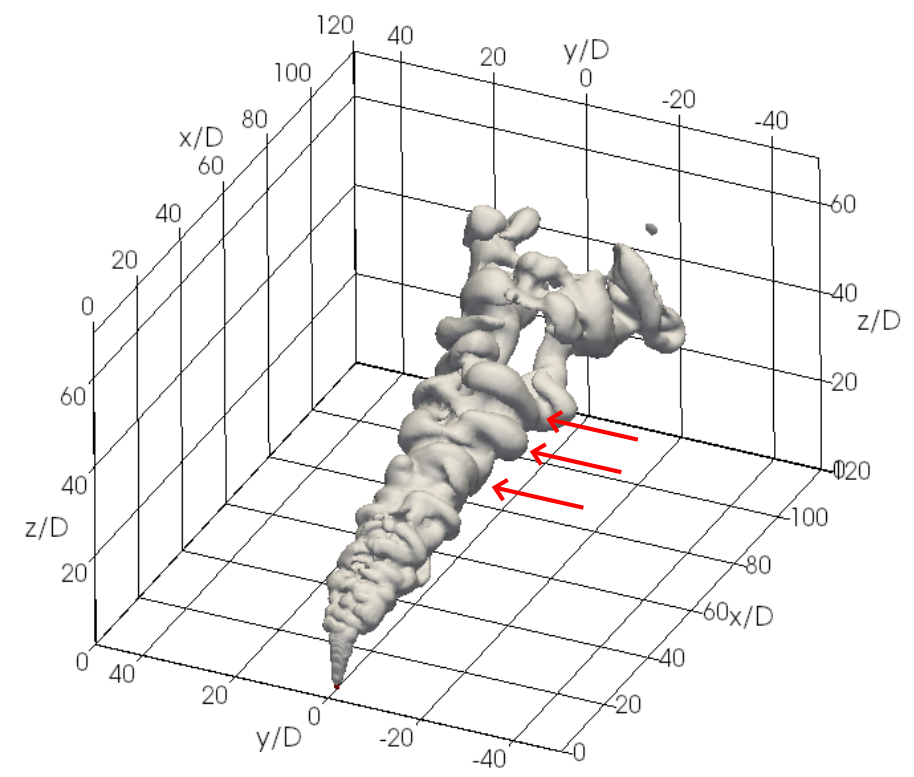

Fig. 16: Contour-plot of instantaneous salinity $s_{j} / s_{j 0}=0.01$ at final time. Red arrows indicate the sausage-like turbulent structures, see section 6.3.

notice that $u_{x}$ tends to the cross-flow values $u_{c f}$ at the centre, except at the level $x / D=10$ where a stagnant point is present as previously underlined. The vertical velocity reaches higher values (more than double with respect to initial condition) in the plane near the nozzle and close to the centre $y / D=0$ (maximum value $x / D=10$ and $y / D=0.1$ ). At this level, it also assumes slightly negative values because of the CVP rotating flow. Moreover, $u_{z}$ experiences a small deflection in the correspondence of the centre, possibly due to the presence of the stagnation point. Downstream, it is more intense in the central zone where it maintains values similar to the initial plume velocity $u_{j 0}$.

\section{Second order statistics and turbulent structures}

The instantaneous structures and the turbulence features of the plume are here studied. The fluctuating quantities are obtained as $\psi^{\prime}=\psi-\langle\psi\rangle(\psi$ is a generic variable), while the root main square (RMS) is computed as $[\psi]_{r m s}=\left\langle\psi^{\prime} \psi^{\prime}\right\rangle^{1 / 2}$. The quantities are made non-dimensional using the scale quantities discussed in section 2.2 .

\subsection{Instantaneous fields}

In order to get an overview of the plume structure, Figure 16 reports the instantaneous salinity isosurface at final simulation time. Such a figure is discussed with respect to the analyses of the mean isosurface reported in section 5 . 

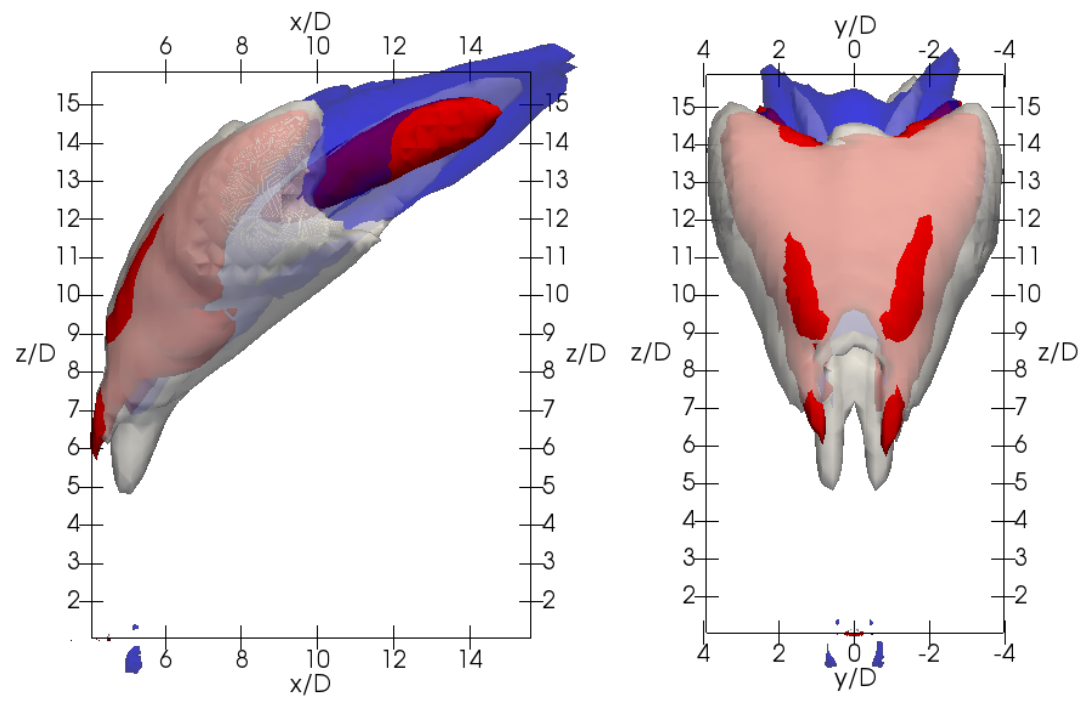

Fig. 17: Isosurfaces of salinity and velocity components RMS: Grey, $\left[s_{j}\right]_{r m s} / s_{j 0}$; Blue, $\left[u_{z}\right]_{r m s} / u_{j 0}$; Red, $\left[u_{x}\right]_{r m s} / u_{c f}$. All the isosurface are computed at the $50 \%$ of the global maximum. Transparency effect is used for better visualisation sake.

The cylindrical shape of the plume just above the nozzle can be considered stable, i.e. the isosurface is just lightly perturbed by instantaneous features. When the entrainment region starts, perturbations arise and become larger and larger moving downstream. In the central region $(40<x / D<80)$ the upper part of the plume is characterised by an irregular surface, where few extrusions of salt water rise from the plume body and increase they dimension going downstream. Such extrusions are identified as turbulent structures, hereafter named sausagelike structures, and analysed in following section section 6.3. When the mean salinity isosurfaces are also visualised, the sausage-like structures seem to encircle the average isosurface from the superior to the lateral side. The tubes of the CVP visible in the average field can be detected also here, even if they are not straight but slightly twisted around the axes. The velocity streamlines within the tube exhibit a helicoidal path, as the result of the superposition of the vortex and the stream-wise motion. As a result, also the tubes delimiting the CVP assume the shape of cylinders twisted around the axes. Near the outflow $(x / D>80)$ the plume becomes more jagged and some patches of salt water separate from the main plume. This part of the domain coincides with the buoyancy-free region, thus it is not considered in the discussion hereafter.

\subsection{Second order statistics}

The RMS of the resolved scalar, stream-wise and spanwise velocity components are discussed. The maximum values reached all over the computational domain are, respectively: $\left[s_{j}\right]_{r m s} / s_{j 0} \leq 0.095,\left[u_{x}\right]_{r m s} / u_{c f} \leq 0.77$ and $\left[u_{z}\right]_{r m s} / u_{j 0} \leq 0.13$. 
Large fluctuations are localised in a zone that appears to be a deflection region where the plume turns from the momentum-buoyancy phase to the fully entrainment one. Figure 17 shows the isosurfaces of the aforementioned RMS quantities; they are computed at a value equal to the $50 \%$ of the maximum reached by the variable. Hence, they contain the most energetic turbulence motion. They are contained within by a parallelepiped with dimensions: $5<x / D, z / D<17$ and $-4<y / D<4$. As expected, the $u_{x}$ RMS is higher in the front part of the plume and in correspondence of the CVP centre; while $u_{z}$ RMS is higher in the reartop part because of the upward flow. It is worth noting that near the nozzle the values are lower. They increase at approximately the height of $z_{B}$ and become substantially higher slightly under $z^{*}$. In this zone the plume is strongly deflected from vertical to horizontal direction and the cross-flow overcomes the momentumbuoyancy initial flow. Later downward, the fully entrainment region begins.

Figure 18 reports the RMS of the same quantities over a vertical plane $x / D=$ 20. The other planes in Figure 13 are not discussed in this contest because they exhibit similar features among them. The salinity RMS peaks around the central core of the CVP (delimited by the five-per-cent salinity contour-plot) where the salt gradient is higher, while it is more diffuse at the top of the plume. The higher values of stream-wise velocity RMS are mainly concentrated at top of the CVP core: here the interaction between plume and cross-flow is more effective. There is a column of low RMS values that goes from the plume to the bottom wall. This region is characterised by the presence of wake vortices that are discussed in next section section 6.3. The vertical velocity RMS has a lower intensity with respect to the other two components, and has local maximum in the central-top part of the CVP tube.

For the sake of completeness, also the RMS profiles along selected horizontal lines are displayed in Figure 19. The line at $x / D=10$ is not smooth because of the high level of fluctuations, hence it is not reported. We can notice that salinity RMS at level $x / D=20$ peaks in two points, approximately correspondent to the edge of the CVP tube. Downstream, the profiles become smoother. The $u_{x}$ RMS exhibits a similar behaviour, while the $u_{z}$ RMS peaks in the centre of the CVP tube. Moving in $x$-direction, the local maximum of the profiles is reached at lower $y / D$.

\subsection{Turbulence structures}

The turbulent features of non-buoyant jet in cross-flow case have been widely studied in literature. Fric and Roshko [18] identified four main large-scale coherent structures: shear layer vortices, horse-shoe vortices, wake vortices and counterrotating vortex pair. Yuan et al. [45] performed an accurate analysis of the initial jet phase, dominated by the initial momentum, pointing out the presence of hanging vortices at the rear of the jet. It is underlined that the structures appearing near the nozzle produce several effects in the downstream behaviour of the jet. In this concern, the origin of the CVP has been particularly debated. It has been suggested that they can be generated either by structures like the ring vortices that appear in absence of cross-flow, or by the hanging vortices (see Fröhlich et al. $[20])$. 

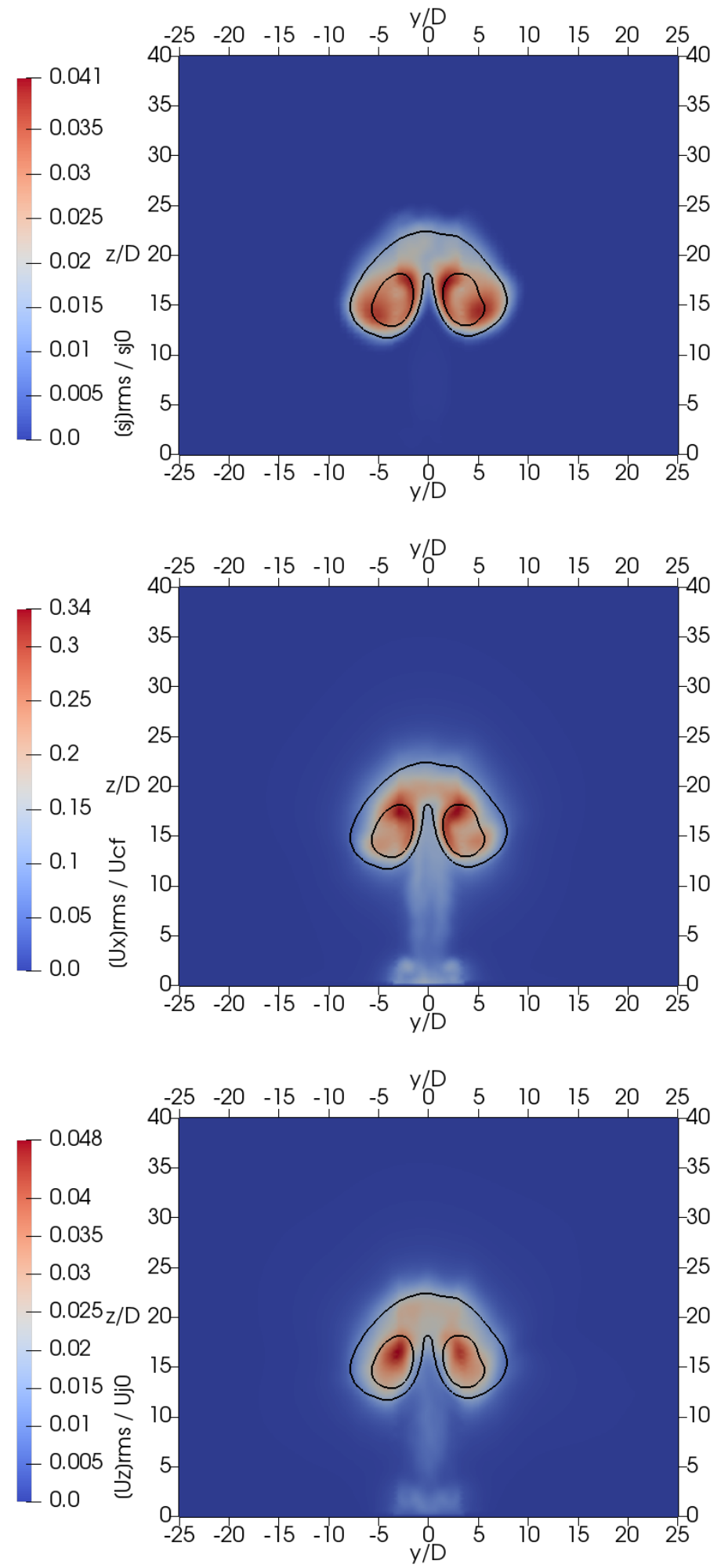

Fig. 18: Non-dimensional mean RMS of salinity, stream-wise velocity and vertical velocity, respectively from the top to the bottom. The visualisation plane is $x / D=20$. The average salinity one-per-cent and five-per-cent counter-lines (as in Figure 13) are also reported. 


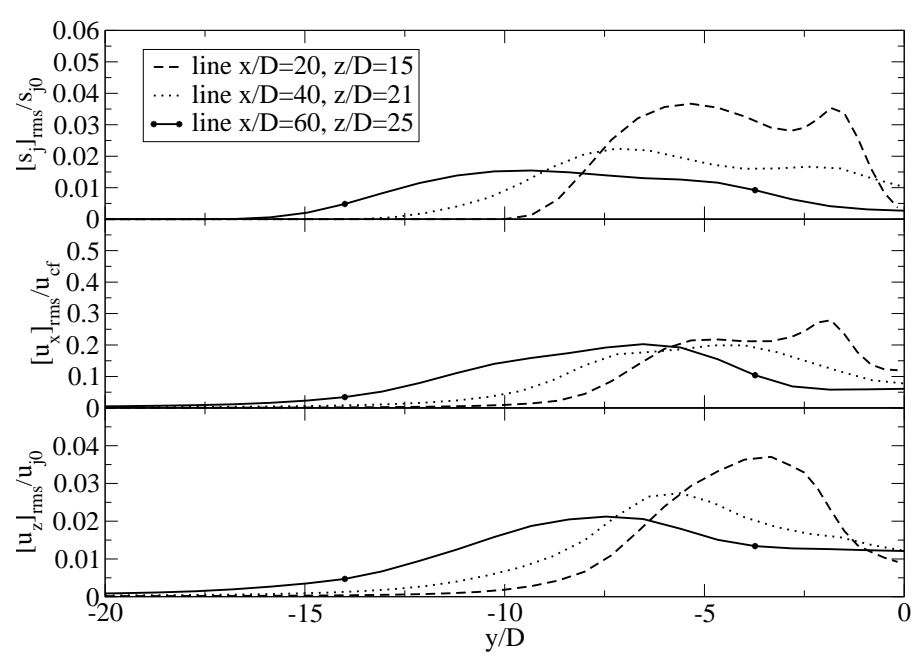

Fig. 19: Profiles of non-dimensional salinity, stream-wise and vertical velocity RMS at three selected lines in spanwise direction as in Figure 15.

The analysis of turbulent features is usually performed by studying the vorticity and pressure isosurface [45] or pressure fluctuation isosurface [12]. The Q-criterion is here preferred to the above-mentioned ones after the analyses of Dubief and Delcayre [15]. Such method consists in the visualisation of the isosurfaces of the second invariant of velocity gradient tensor:

$$
Q=\frac{1}{2}\left(\Omega_{i j} \Omega_{i j}-S_{i j} S_{i j}\right)
$$

where $\Omega_{i j}=\left(\partial u_{i} / \partial x_{j}-\partial u_{j} / \partial x_{i}\right) / 2$. This can be interpreted as the balance between rotational rate and strain rate; hence, the isosurfaces of positive values are qualified to be vortices delimiter.

Figure 20 displays the isosurface for $Q=0.75$ from two different perspectives. Several structures (also detected in the JICF case) can be identified, along with the already mentioned sausage-like structures. For the sake of completeness, they are briefly recalled:

- The wake vortices are clearly visible just behind the plume. Columns of vertical vortices arise from the bottom wall and arrive till the groove between the two branches of the plume. They almost disappear downstream after $x / D=40$.

- The shear layer vortices are visible in the front side of the plume. At the deflection region they assume the shape of annular rings which encompass the top part of the plume. The effect of these perturbations is to ripple the salinity isosurface, as can be seen also in Figure 16.

- The fully entrainment region is characterised by thicker structures. A comparison with the salinity isosurface demonstrates that such elements are localised in the core of the sausage-like structures visible in Figure 16.

- The CVP are represented by the two large twisted tubes that tend to separate going downstream. They exhibit the typical helicoidal shape already discussed. 

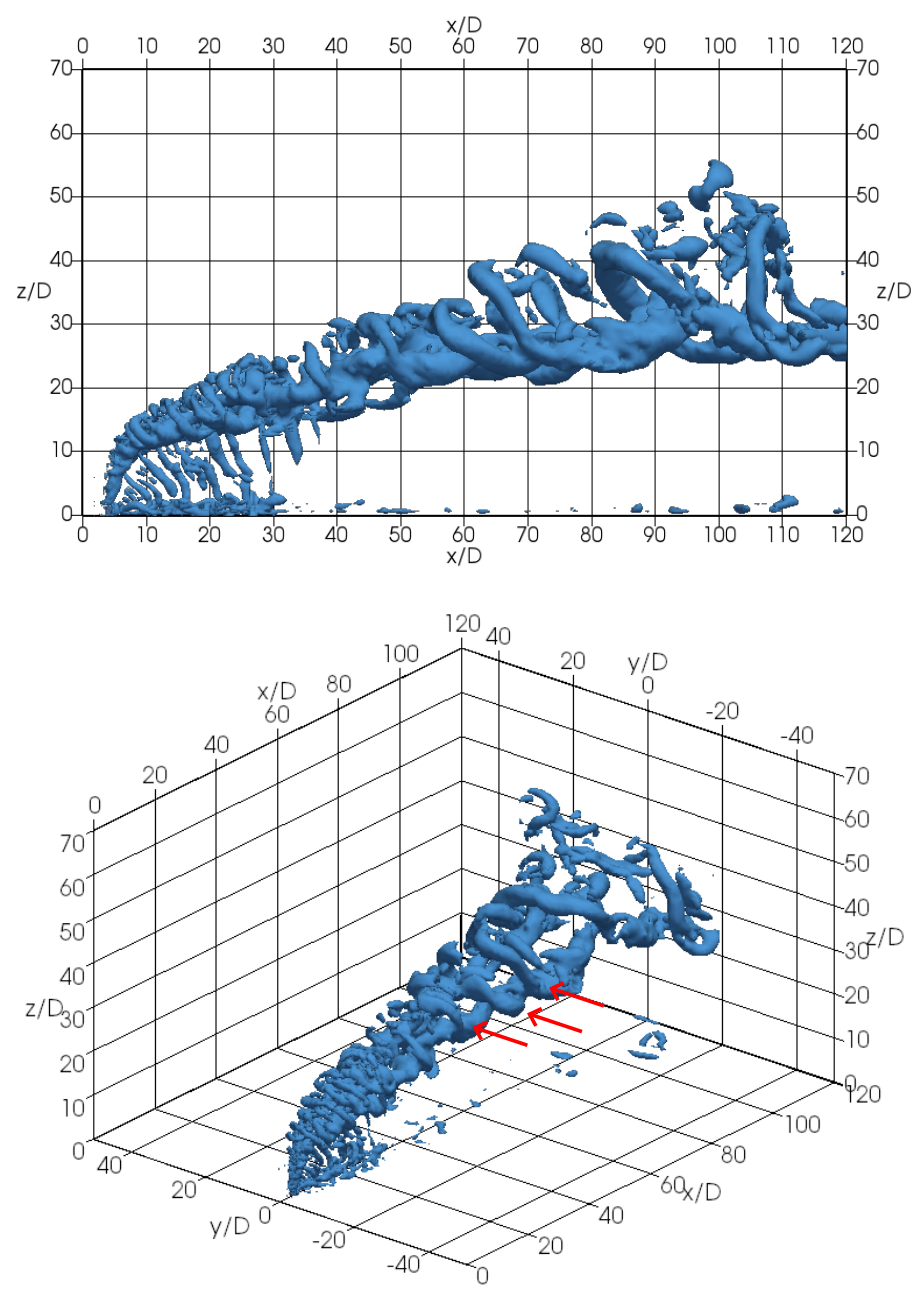

Fig. 20: Isosurface for $Q=0.75$ from two different perspectives. From the top to the bottom, respectively: lateral view (along $y$-direction) and three-dimensional perspectives. Red arrows indicate the sausage-like turbulent structures.

No particular structure is visible just above the nozzle, where both mean and instantaneous salinity isosurfaces are smooth. It is possible that the upward flow is sufficiently intense with respect to the cross-flow not to be substantially altered.

The sausage-like structures are now described with the help of the diagram in Figure 21. They appear to be vortex tubes that encircle the mean counterrotating vortices from the top to the external lateral side. This shape recalls a semi-circular sausage, from which the name comes. Three of these structures are visible in Figure 16, and their core is highlighted by the Q-isosurface in Figure 20. The sausage-like structures origin from the shear layer vortices and evolve in the 


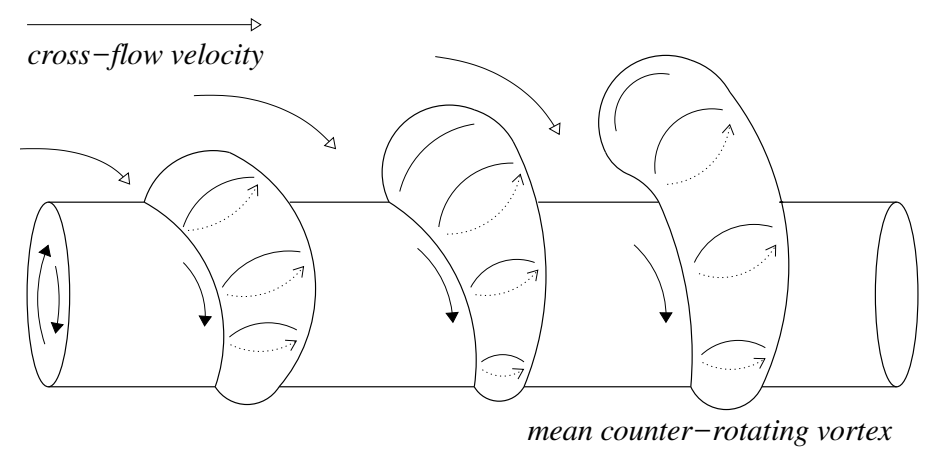

Fig. 21: Schematic picture of the sausage-like structures appearing in the region $40<x / D<80$; see also Figure16 and 20. The central tube represents one of the CVP. Empty arrows: cross-flow direction; solid arrows: direction of vortex rotation; dashed arrows: buoyancy force direction.

entrainment region $(40<x / D<80)$, where the shear stress decreases as a consequence of the plume deflection in stream-wise direction. The shear reduction inhibits the formation of new vortices, while that ones coming from the deflection region becomes less energetic and increase in size. The result is that the upper part of the plume is corrugated, characterised by crests and valleys. At this stage, three players combine their actions: the counter-rotating vortex tends to elongate and stretch the structures on its surface, from top to external lateral side; the cross-flow canalises in the valleys, generating pockets of fresh water and inducing the separation of structures from the counter-rotating vortex; the buoyancy force supports the internal rotational motion with an upward velocity component. Moving downstream, the sausage-like structures separate from the mean plume body, eventually disappearing in the top part of the domain.

Here we make a difference between the shear layer vortices triggered in the deflection region, and the larger sausage-like structures which appear in the entrainment region. They can also be considered as two phases of a single structure that evolves in time and space, but there are some differences that can justify this choice: the former surround the entire top edge of the plume, are thin and develop in a region characterised by high shear rate. The latter cover the top surface and part of the lateral surface of the plume, they are substantially thicker and characterised by less energetic shear.

In order to investigate on the origin of the CVP, the velocity streamlines above the nozzle are scrutinised. Figure 22 displays the averaged velocity streamlines in the proximity of the nozzle, coloured by $\left\langle u_{y}\right\rangle$. In agreement with the analysis proposed by Denev et al. [12], the plume above the nozzle acts as an obstacle for the cross-flow and generates a recirculation region in the plume rear. Differently from the case of solid obstacle, the plume pulls upward the fluid producing a vertical velocity that combines with the rotating motion of the recirculation vortices and gives rise to helicoidal vertical flows. Such dynamics can be clearly detected in the red streamlines in the leeward side of the plume. The vertical deflection is also revealed by the streamlines that start near the nozzle and that develop upward. 


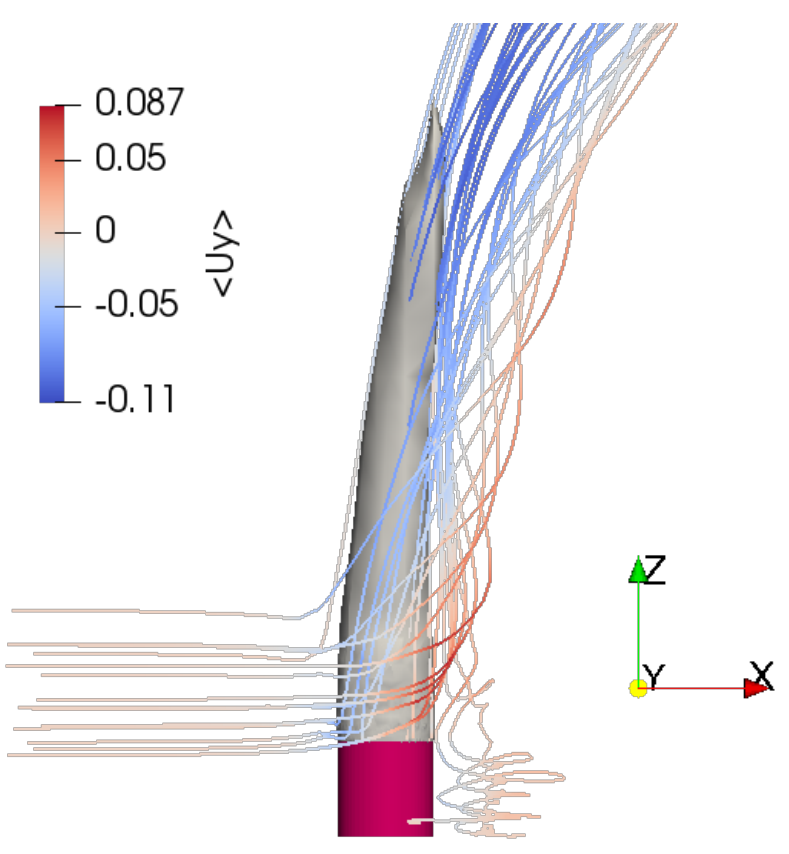

Fig. 22: Streamlines in the proximity of the nozzle (marked in dark pink) coloured by the mean spanwise velocity component. The isosurface $\left\langle s_{j}\right\rangle / s_{j 0}=0.5$ are also reported in grey.

In the deflection region, when the initial momentum-buoyancy force decreases, the cross-flow deflects the plume and at the same time the helicoidal flows drive freshwater from the leeward side toward the plume. This generates the groove of freshwater between the CVP. The counter-rotating helicoidal flows start to separate and in the entrainment region evolve in the CVP.

\section{Comparison with the non-buoyant case}

The effects of buoyancy force are investigated comparing the BJCF case with an additional simulation where the buoyancy force is switched off. This second simulation is performed using the same settings as the previous one, but salinity is a passive scalar. For the sake of simplicity, we refer to the buoyant jet as the plume and to the non-buoyant jet as the jet. From a practical point of view, the simulation is representative of a jet of water containing a contaminant in a crossflow of ambient water at the same temperature and salinity.

First, the mean field is analysed. Figure 23-top shows the jet trajectory; as expected, the jet rises sensibly less than the plume: after the point $x / D>10$ the two trajectories separate and the difference between them increases constantly. For the JICF case, there is not a clear consensus regarding the semi-empirical formula for trajectory; see Mahesh [32]. An empirical formula that fits the present data 


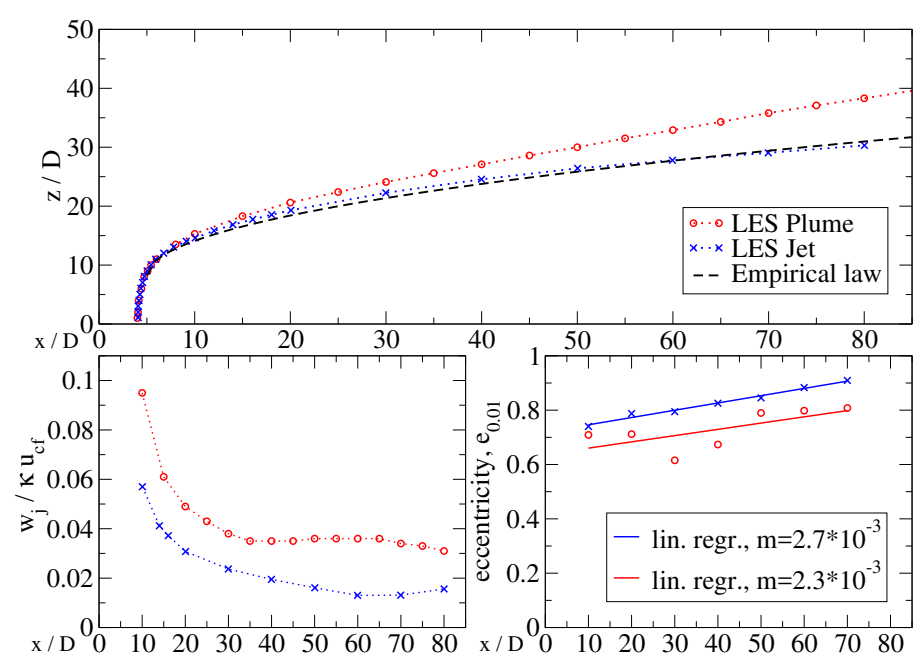

Fig. 23: Comparison between buoyant and non-buoyant jet in cross-flow, respectively labelled plume and jet. Top: simulated mean trajectories at the vertical centre plane and empirical profile $z_{j e t}$. Bottom left: non-dimensional vertical velocity component. Bottom right: eccentricity for the one-per-cent isosurface and linear regression line.

is here proposed, although we are well aware that its exhaustive validation would need successive studies with different forcing conditions. Rearranging the terms of equation (9) for the plume height $z_{j}$, one obtains:

$$
z_{\text {plume }}=\text { const } D\left(\frac{u_{j 0}}{u_{c f}}\right)^{\frac{1}{3}}\left(\frac{u_{b}}{u_{c f}}\right)^{\frac{2}{3}}\left(\frac{x_{j}}{D}\right)^{\frac{2}{3}},
$$

where the value of the constant does not depend on flow parameters. Hence, the trajectory height is proportional to the non-dimensional stream-wise coordinate through the nozzle diameter and the ratio of the vertical velocities to the crossflow velocity. By analogy, a possible empirical profile for the jet trajectory can be expressed by:

$$
z_{\text {jet }}=\text { const } D\left(\frac{u_{j 0}}{u_{c f}}\right)\left(\frac{x_{j}}{D}\right)^{\xi}=\frac{1}{3} D \kappa\left(\frac{x_{j}}{D}\right)^{\frac{1}{2}}
$$

where const $=1 / 3$ and $\xi=1 / 2$ are found to be the values that best fit the simulation data. Despite this expression is derived by simple analogy and, moreover, it is not proven to have general validity, it is interesting to notice that the jet trajectory follows the law $z_{j e t} \propto x^{\frac{1}{2}}$, while the plume follows $z_{\text {plume }} \propto x^{\frac{2}{3}}$. This result is in agreement with the study of Hasselbrink and Mungal [24] on JICF.

Figure 23-bottom-left reports the non-dimensional vertical velocity component at the trajectory height. On average, the jet velocity is $5 \%$ lower than the plume one in the bent-over region.

Figure 23-bottom-right presents the eccentricity relative to the one-per-cent salinity isosurface (see definition in section 5 ). The jet eccentricity is $10 \%$ higher 


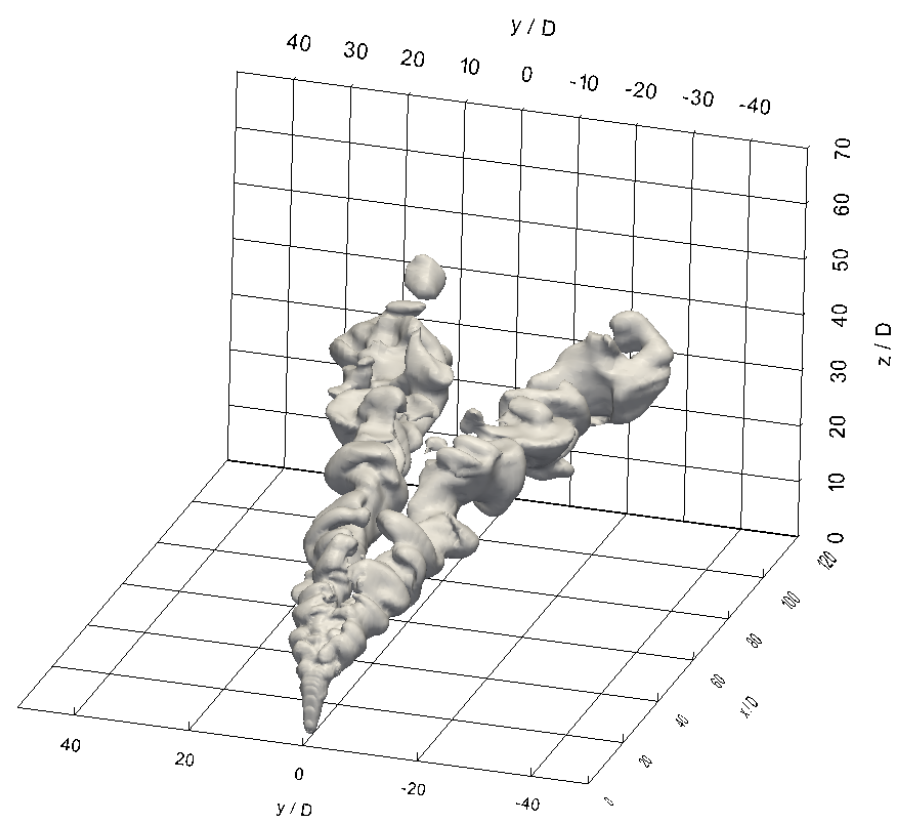

Fig. 24: Isosurface of instantaneous salinity $s_{j} / s_{j 0}=0.01$.

than plume eccentricity and it increases with the same slope. Also, the radius $r_{j}$ has been checked, but the profile does not exhibit a substantial difference in the two cases and it is not here reported. This can be explained by the fact that the jet CVP tends to separate more rapidly, but they are less extended in vertical direction; thus, the jet horizontal ratio $R_{h}$ is larger while the vertical one $R_{v}$ is smaller with respect to the plume case. The result is a similar value of $r_{j}$ but a larger eccentricity in the jet case. The salinity concentration $s_{j}$ profiles do not show relevant differences between cases: the jet salinity has just slightly lower values in the entrainment region. Hence, the buoyancy contribution to mixing appears limited.

Figure 24 shows the instantaneous one-per-cent salinity isosurface. The three main differences between the jet and the plume, pointed out in the above discussion, can be detected: the jet trajectory is less high, salinity isosurface is less extended in vertical direction, and the gap between the CVP increases by a higher rate along the stream-wise direction. Also, the mean one-per-cent salinity isosurface (not reported) clearly exhibits these peculiarities. It is worth noting that the sausage-like structures are not visible: few extrusions of salty water are detectable at the beginning of the entrainment region, but later downstream they do not assume the typical semi-circle tube shape. Consistently, the Q-isosurface does not point out the presence of such turbulent features in the region $40<x / D<80$. Buoyancy appears to play a crucial role in the formation of sausage-like structures, supporting the internal rotational motion against the cross-flow. In the absence of 
this force, the turbulent energy is rapidly dissipated by the cross-flow and salinity extrusions are passively transported.

\section{Conclusions}

Large-eddy simulations of buoyant (plume) and non-buoyant jet in cross-flow are performed adopting the dynamic Lagrangian sub-grid scale model for momentum and scalar equations. One of the experimental plume cases reported in Fan $[16]$ is reproduced and successfully validated against experimental data and semiempirical relations. Such a case is characterised by Froude number $F=10$ and velocity ratio $\kappa=8$ (Reynolds number based on the plume velocity $R e=8200$ ). Subsequently, the first and second order statistics of the fluid flow are analysed and the $Q$-criterion is used to study turbulent structures.

The main results of the study are here summarised. The plume evolution is composed of three main regions:

- momentum-buoyancy region: just above the nozzle it extends approximately up to $z / D=8$, where the plume is injected in the cross-flow. It is characterised by strong vertical motion, low level of scalar and velocity fluctuations, relative small turbulent structures on the plume edge. The plume exhibits an almost steady vertical cylinder-like shape.

- deflection region: here the plume is deflected by the cross-flow, approximately in a three-dimensional region extending $8<x / D, z / D<17$ and $-4<y / D<4$. The initial momentum and buoyancy force are perturbed by the interaction with the cross-flow, which deflects the plume trajectory from vertical toward the horizontal direction. The plume enlarges in the cross-stream directions in an anisotropic way, being larger in the horizontal than in vertical direction. Such region presents the highest energetic turbulence (quantified by means of scalar and velocity root main squares); the shear layer vortices are clearly visible at the superior plume boundary and are responsible for most of the fluctuating field.

- entrainment region: here the cross-flow overcomes the initial momentum-buoyancy; this region extends downstream beyond $x / D>17$. The plume here splits into two branches, the motion intensity is attenuated and it is mainly dominated by the counter-rotating vortex pair. At the plume top boundary, the sausagelike turbulent structures develop, separated by pockets of almost zero scalar concentration. These structures appear to be generated by buoyancy force that supports the internal rotational motion, supplying energy against the dissipative interaction with the cross-flow.

In order to estimate the effects of buoyancy force, a simulation sharing the same features that the buoyant one but without gravitational effects was performed. The comparison between buoyant (plume) and non-buoyant (jet) case shows that: (i) the trajectory of the jet is sensibly lower and goes like $x^{\frac{1}{2}}$, different from the $x^{\frac{2}{3}}$ behaviour of the plume; (ii) the vertical velocity is about $5 \%$ lower than in the plume case; (iii) the eccentricity is $10 \%$ higher; (iv) the CVP diverges with higher rate along the stream-wise direction. The jet in cross-flow does not exhibit the sausage-like turbulent structures. 
The origin of the counter-rotating vortex pair is investigated. In agreement with $[45,12]$, it has been identified in the upward helicoidal flow that arises at the lateral leeward side of the plume, near the nozzle area. This flow is a combination of vertical plume entrainment and recirculation motion behind the plume.

Finally, an empirical expression for the jet trajectory is derived by analogy with the semi-empirical formula for plumes. The simulation data show that the trajectory height increases as the square root of the stream-wise coordinate, in accordance with literature results. The general validity of such expression is not proven and a complete validation requires additional research.

Acknowledgements This work was supported by Regione Friuli-Venezia Giulia - DITENAVE - Progetto "CFD open source per opera morta - COSMO" n. CUP J94C14000090006.

\section{References}

1. V. Armenio and S. Sarkar. An investigation of stably stratified turbulent channel flow using large-eddy simulation. J. Fluid Mechanics, 459:1, 2002.

2. V. Armenio. Mathematical modeling of stratified flows. In: V. Armenio and S. Sarkar (eds) Environmental Stratified Flows. CISM International Centre for Mechanical Sciences (Courses and Lectures), 479, 2005. doi: doi.org/10.1007/ 3-211-38078-7_1.

3. R. Camussi, G. Guj, and A. Stella. Experimental study of a jet in a crossflow at very low reynolds number. Journal of Fluid Mechanics, 454:113144, 2002.

4. D. Cavar and K. E. Meyer. Les of turbulent jet in cross-flow: Part 1 a numerical validation study. International Journal of Heat and Fluid Flow, 36 (Supplement C):18 - 34, 2012. doi: doi.org/10.1016/j.ijheatfluidflow.2011.12. 009.

5. V. H. Chu and M. B. Goldberg. Buoyant forced-plumes in cross flow. Journal of the Hydraulic Division, 100, 1974.

6. C. Cintolesi, A. Petronio, and V. Armenio. Large eddy simulation of turbulent buoyant flow in a confined cavity with conjugate heat transfer. Physics of fluids, 27, 2015. doi: dx.doi.org/10.1063/1.4930815.

7. C. Cintolesi, A. Petronio, and V. Armenio. Large-eddy simulation of thin film evaporation and condensation from a hot plate in enclosure: First order statistics. International Journal of Heat and Mass Transfer, 101:1123, 2016. doi: dx.doi.org/10.1016/j.ijheatmasstransfer.2016.06.006.

8. C. Cintolesi, A. Petronio, and V. Armenio. Large-eddy simulation of thin film evaporation and condensation from a hot plate in enclosure: Second order statistics. International Journal of Heat and Mass Transfer, 115:410-423, 2017. doi: dx.doi.org/10.1016/j.ijheatmasstransfer.2017.08.043.

9. P. Cunningham, S. Goodrick, Y. M. Hussaini, and R. Linn. Coherent vortical structures in numerical simulations of buoyant plumes from wildland fires. International Journal of Wildland Fire, 14:61-75, 2004. doi: doi.org/10.1071/ WF04044.

10. L. de Wit and C. van Rhee. Testing an improved artificial viscosity advection scheme to minimise wiggles in large eddy simulation of buoyant jet in crossflow. Flow, Turbulence and Combustion, 92, 2014. 
11. L. de Wit, C. van Rhee, and G. Keetels. Turbulent interaction of a buoyant jet in cross-flow. Journal of Hydraulic Engineering, 140, 2014.

12. J. A. Denev, J. Fröhlich, and H. Bockhorn. Large eddy simulation of a swirling transverse jet into a crossflow with investigation of scalar transport. Physics of Fluids, 21, 2009. doi: dx.doi.org/10.1063/1.3054148.

13. B. J. Devenish, G. G. Rooney, H. N. Webster, and D. J. Thomson. The entrainment rate for buoyant plumes in a crossflow. Boundary-Layer Meteorology, 134, 2010.

14. P. E. Dimotakis. Mixing transition in turbulent flows. Journal of Fluid Mechanics, 409:69-98, 2000.

15. Y. Dubief and F. Delcayre. On coherent-vortex identification in turbulence. Journal of Turbulence, 1:11, 2000. doi: 10.1088/1468-5248/1/1/011.

16. Loh-Nien Fan. Turbulent buoyant jets into stratified or flowing ambient fluids. Ph.D. thesis, California Institute of Technology, 1967.

17. H. Fischer, J. List, C. Koh, J. Imberger, and N. Brooks. Mixing in inland and coastal waters. Academic Press, 1979.

18. T. F. Fric and A. Roshko. Vortical structure in the wake of a transverse jet. Journal of Fluid Mechanics, 279:147, 1994. doi: 10.1017/S0022112094003800.

19. J. Fröhlich, C. P. Mellen, W. Rodi, L. Temmerman, and M. A. Leschziner. Highly resolved large-eddy simulation of separated flow in a channel with streamwise periodic constrictions. Journal of Fluid Mechanics, 526:1966, 2005. doi: $10.1017 /$ S0022112004002812.

20. J. Fröhlich, J. A. Denev, and H. Bockhorn. Large eddy simulation of of a jet in crossflow. ECCOMAS, 24-28 July 2004.

21. S. J. Gaskin. Single Buoyant Jets in a Crossflow and the Advected Line Thermal. Ph.D. thesis, University of Canterbury, Christchurch, New Zealand, 1995.

22. M. Germano, U. Piomelli, P. Moin, and W. Cabot. A dynamic subgrid-scale eddy viscosity model. Phys. Fluids, A3:1760, 1991.

23. D. D. Gray and A. Giorgini. The validity of the boussinesq approximation for liquids and gases. Int. J. Heat Mass Transfer, 19:545-551, 1976. doi: 10.1016/0017-9310(76)90168-X.

24. E. F. Hasselbrink and M. G. Mungal. Transverse jets and jet flames. part 1. scaling laws for strong transverse jets. Journal of Fluid Mechanics, 443:125, 2001. doi: 10.1017/S0022112001005146.

25. P. Huq and E.J. Stewart. A laboratory study of buoyant plumes in laminar and turbulent crossflows. Atmospheric Environment, 30(7):1125 - 1135, 1996. doi: 10.1016/1352-2310(95)00335-5.

26. H. Jasak, H.G. Weller, and A.D. Gosman. High resolution nvd differencing scheme for arbitrarily unstructured meshes. Int. J. Numer. Meth. Fluids, 31: 431-449, 1999.

27. E. Keramarisa and G. Pechlivanidis. The behaviour of a turbulent buoyant jet into flowing environment. International Conference on Efficient and Sustainable Water Systems Management toward Worth Living Development, 2nd EWaS 2016, 162:120-127, 2016.

28. B.E. Launder and D.B. Spalding. The numerical computation of turbulent flows. Computer Methods in Applied Mechanics and Engineering, 2:263-289, 1974.

29. J. W. Lavelle. Buoyancy-driven plumes in rotating, stratified cross flows: Plume dependence on rotation, turbulent mixing, and crossflow strength. 
Journal of Geophysical Research: Oceans, 102(C2):3405-3420, 1997. doi: 10.1029/96JC03601.

30. J. Hun-Wei Lee and V. Chu. Turbulent Jets and Plumes. Springer - Kluwer Academic Publisher, 2003.

31. F. Ma, M. Satish, and M. R. Islam. Large eddy simulation of thermal jets in cross flow. Engineering Applications of Computational Fluid Mechanics, 1(1): 25-35, 2007. doi: 10.1080/19942060.2007.11015179.

32. K. Mahesh. The interaction of jets with crossflow. Annual Review of Fluid Mechanics, 45(1):379-407, 2013. doi: 10.1146/annurev-fluid-120710-101115.

33. M. B. Meftah, P. Davies, D. Malcangio, and M. Mossa. Turbulence of vertical round buoyant jets in a crossflow. In Proc. River Flow, volume 2, pages 11671174, 2004.

34. C. Meneveau, T.S. Lund, and W.H. Cabot. A lagrangian dynamic subgridscale model of turbulence. J. Fluid Mechanics, 316:353, 1996.

35. Z. M. Moussa, J.W. Trischka, and S. Eskinazi. The near-field in the mixing of a round jet with a cross-stream. Journal of Fluid Mechanics, 80:49-80, 1977.

36. P. J. Oliveira and P. I. Issa. An improved piso algorithm for the computation of bouyancy driven flows. Numerical Heat Transfers, Part B. Fundamentals, 640:473, 2001.

37. U. Piomelli. Large-eddy and direct simulation of turbulent flows. CFD2001 9th Conférence Annuelle de la Société Canadienne de CFD, 2001.

38. S.B. Pope. Turbulent Flows. Cambridge University Press, 2000.

39. P. Sagaut. Large eddy simulation for incompressible flows. An introduction. Springer, 2000.

40. S. H. Smith and M. G. Mungal. Mixing, structure and scaling of the jet in cross-flow. Journal of Fluid Mechanics, 357:83-122, 1998.

41. B. van Leer. Towards the ultimate conservative difference scheme. v. a secondorder sequel to godunov's method. Journal of Computational Physics, 32(1): 101-136, 1979. doi: 10.1016/0021-9991(79)90145-1.

42. S. J. Wright. Mean behavior of buoyant jets in a crossflow. Journal of the Hydraulic Division, 103, 1977.

43. S. J. Wright. Buoyant jets in density-stratified crossflow. Journal of Hydraulic Engineering, 110:643-656, 1984.

44. L. L. Yuan and R. L. Street. Trajectory and entrainment of a round jet in crossflow. Physics of Fluids, 10(9):2323-2335, 1998. doi: 10.1063/1.869751.

45. L. L. Yuan, R. L. Street, and J. H. Ferziger. Large-eddy simulations of a round jet in crossflow. Journal of Fluid Mechanics, 379:71-104, 1999. doi: 10.1017/S0022112098003346. 